$1^{\text {st }}$ Revision of the Manuscript: Confidential

\title{
Chipping and Crushing Mechanisms in Orthogonal Rock Cutting
}

\author{
Demeng Che ${ }^{1}$, Wu-Le Zhu, Kornel F. Ehmann \\ Department of Mechanical Engineering \\ Northwestern University \\ 2145 Sheridan Road, Evanston, Illinois, USA, 60208
}

\begin{abstract}
The understanding of rock removal mechanisms, as one of the essential elements for advancing the cutting performance of polycrystalline diamond compact (PDC) cutters, is still incomplete. This paper is seeking to formulate a cutting theory to analytically explain how rock interacts with PDC cutters in a simple cutting configuration - linear cutting. In the proposed approach, a rock crushing zone is introduced at the rock-cutter interface beneath the cutter's rake face, namely the Tip Crushing Zone (TCZ), which, as a destruction kernel, is responsible for both the crushing and chipping phenomena occurring in the cutting process. The physical aspects of these two disparate chip formation mechanisms are discussed. Moreover, closed form expressions for the cutting and thrust forces are derived based on the equilibrium of moments and on the minimum chipping energy principle. To evaluate the force model, comprehensive linear rock cutting tests were conducted on a newly developed rock cutting facility. Both the chip formation phenomena and force responses are in good agreement with the newly proposed theory.
\end{abstract}

\section{Keywords}

Rock cutting · Polycrystalline diamond compact (PDC) cutter · Cutting mechanics · Analytical model $\cdot$ Cutting theory $\cdot$ Rock removal mechanism

\section{Introduction}

Rock shearing is a cutting process in which a cutting tool is dragged against a rock formation along a certain trajectory to facilitate rock material removal. Different trajectories are used in different removal applications. For instance, in modern oil/gas well drilling processes, polycrystalline diamond compact (PDC) cutters advance along a spiral trajectory as shown in Fig. 1. The introduction of the shearing action into the process has highly improved the drilling efficiency in comparison to the traditional gouging and crushing actions [1]. On the other hand, the shearing action makes PDC cutters intrinsically sensitive to the material properties of the drilled rock formations. Catastrophic damage such as cutter delamination and chipping commonly exist in drilling of extremely hard and abrasive rock formations [2]. Therefore, a comprehensive understanding of the rock-cutter interactions in shearing is highly needed which, if obtained, could offer basic guidelines for improving the cutting performance of PDC cutters and preventing catastrophic damage of the cutters [3]. Such a complete knowledge, however, is still missing due to the complexity of the shearing action and the rock's material properties, such as its inhomogeneity, dilatancy, and pressure-dependent failure behavior $[\underline{4}, \underline{5}]$.

\footnotetext{
${ }^{1}$ Corresponding author. Tel: +1 (847) 467-0712; Fax: +1 (847) 491-3915

Email address: dche@u.northwestern.edu
} 


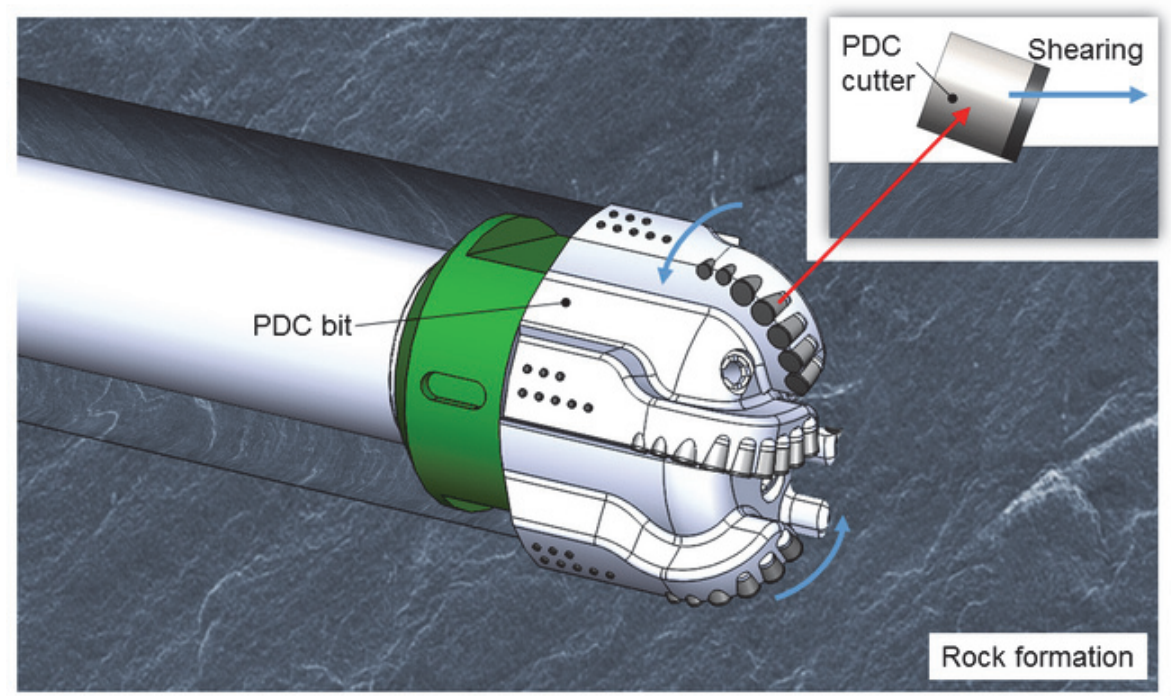

Fig. 1 Rock drilling performed with a commercial PDC bit.

In light of the above, in this paper an attempt will be made to formulate a novel cutting theory which is aimed at explaining rock removal mechanisms from a physical point of view that is rooted in experimental observations and derive corresponding force prediction relationships. Before going into the theory, however, a literature review is given to overview the tremendous efforts done in rock cutting theories in the past and explain the rationale for the approach proposed in this paper.

Although rock removal mechanisms have been explained in various ways, there are two major failure modes widely considered to be involved in the cutting processes, i.e., the crushing and chipping modes. The crushing mode creates highly fractured and inelastically deformed rock, while the chipping mode initiates and propagates cracks to form big chips []ㅡ. Although some researchers have proposed that rock fails in a single failure mode $[\underline{7}, \underline{8}]$, a crushing-chipping hybrid failure mode has been widely accepted. Fine particles and large chips were found to be alternatively produced in a series of linear cutting tests conducted in 1956 [9]. Later on, typical rock chips were found to include an apparent crushed and a powdery zone in another linear rock cutting test [10]. According to these facts, a crushed zone of rock, namely the destruction kernel, was proposed to initiate/induce the formation of chip segments in a continuous shearing process $[\underline{6}, \underline{11}]$, which can be referred to as the crushing-induced chipping mode. A similar rock failure mode was also mentioned in some other researchers' work [12-14]. In the current work the crushing-induced chipping failure mode of rock failure will be adopted given the overwhelming experimental evidence in its favor. Further justification for this choice is given below.

The chipping of rock is an extremely complex fracture process whose fundamental mechanisms have been studied over the past five decades but a clear consensus has not yet emerged. Some researchers proposed that chipping is caused by tensile breakage $[\underline{7}, \underline{8}, \underline{15}, \underline{16}]$ while others believed that the influence of the shear stresses is the intrinsic reasons for chipping [6, 17-21]. Shear stresses have been found to significantly influence the continuous chip formation of metals because of their obvious plastic behavior. On the other hand, rocks are generally observed to fail more like other brittle materials with discontinuous chips. To this end, the current work will be based on the assumption that the tensile stresses govern the rock's chipping process. 
Experimental observations of rock failure processes are still limited to the measurements of the chip size, the recording of chip formation at the macro-scale, and the acquisition of force signals. Among them, force data are the easiest to collect and, therefore, are commonly used to indicate the cutter's performance and evaluate the predictive accuracy of developed rock cutting theories. Many force models have been put forward to predict force responses in rock cutting processes since the 1950s. Some force models were built to relate force/energy data to cutting parameters by curve fitting or regression methods under certain physical assumptions such as linear dependence of the force on cutting depth and a constant friction coefficient [22-25]. Such models, i.e., phenomenological models, could quickly provide accurate force predictions in a certain parameter range and, as such are quite popular in industrial-level applications. However, to some extent, they fail to explain the intrinsic mechanisms of force responses from a physical point of view.

To overcome the limitations of phenomenological models, other researchers attempted to build force models based on the complete stress distributions obtained from solid and fracture mechanics theories [12, 26, 27]. Due to the complex mathematical formulations, closed form solutions for such models are usually difficult to obtain. In many cases, numerical methods are, therefore, needed to solve the built models [28-30]. In response, simplified force models were developed to predict the rock's failure behavior by defining critical stress distributions $[\underline{7}, \underline{17}, \underline{18}, \underline{31}]$. Such models, if properly built, could provide closed form expressions for the force responses with trustful physical meanings. The newly proposed theory and models presented in this paper fall into this category. The idea of such force models originates from Merchant's metal cutting model $[32,33]$ in which the critical shear stresses were assumed to follow a straight line in front of the tool tip. The model was later on modified with a Mohr-Coulomb failure criterion to provide more accurate force response predictions in rock cutting [17, $\underline{18}, \underline{34}]$. However, the assumption of a linear shear plane is not realistic under the conditions of the very limited shearinduced plastic deformations in rock cutting. To alleviate this problem, critical tensile stresses along a curved propagation path were proposed to determine the rock's chipping behavior [ㄱ, 27, 35]. This was done in accordance with the rock's tensile failure behavior in uniaxial compression tests and in correspondence to the big curved chips obtained in coal mining. However, the tiny powder-like chips observed in rock cutting tests could not be explained by such models. More recently, a crushing zone was included in the force models to be responsible for the formation of powder-like chips [29, $\underline{31}]$ but the models failed to predict the force responses when the cutting tool orientation (i.e., the rake angle) was changed. A more detailed literature review of rock cutting theories can be found in the author's previous publications $[\underline{4}, \underline{5}]$.

In response to the above, this paper aims to develop a new cutting theory that is more closely in tune with the observed experimental evidence and rooted in realistic rock removal mechanisms in order to formulate more accurate force predictions. To achieve this goal, a crushing zone, namely the Tip Crushing Zone (TCZ) is defined right beneath the cutter's rake face with a specific stress distribution. The $\mathrm{TCZ}$, as it will be shown, determines the formation of power-like chips in accordance with the experimental observance. A crack that is assumed to initiate at an arbitrary location on the boundary of the TCZ and propagates towards the free surface of the virgin rock will, in turn, explain the observed chipping failure of rock. The force responses are then derived based on the equilibrium of moments applied to the chip and the minimum chipping energy principle. A series of linear rock cutting tests 
conducted on a newly developed rock cutting facility will facilitate the calibration and feasibility assessment of the developed force model.

The organization of this paper is given as follows: Section 2 develops the above-mentioned new cutting theory, followed by an introduction of the newly developed testbed for the linear cutting of rock described in Section 3. Then, the cutting theory will be evaluated in Section 4, by comparing the force predictions with experimental measurements. Several characteristic parameters for this new theory will be discussed in Section 5, followed by the conclusions drawn in Section 6.

\section{Theory}

This section presents a new cutting theory which explains how rock fails in the linear rock cutting process and then provides predictions of the forces exerted by the PDC cutter onto the rock. The ultimate goal of this theory is to reveal the nature of rock failure and then formulate closed form expressions for the two orthogonal force components in rock cutting, namely the cutting and thrust forces, which are defined as force components along and perpendicular to the cutting direction respectively, as shown in Fig. 2. To achieve this goal, necessary assumptions are made to simplify this problem in accordance with realistic cutting conditions, as summarized in Section 2.1. For a better understanding of the theory, Section 2.2 will discuss the derivation of a closed form expression of the cutting force for a simple model. Then, the cutting force model will be naturally extended to a more general model in Section 2.3 with intrinsic similarities to the simple model. Based on the derived general cutting force model, a thrust force model will also be given by defining a new process parameter from a physical perspective, which will be discussed in Section 2.4.

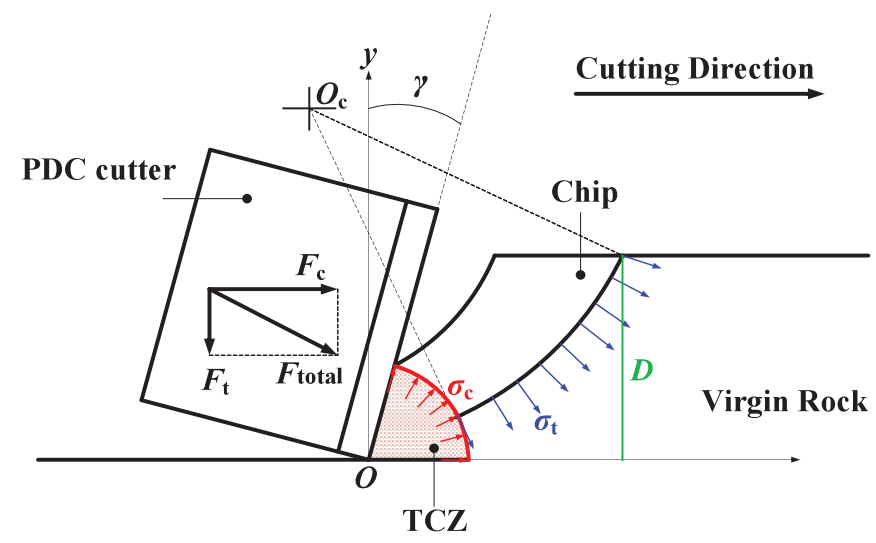

Fig. 2 Schematics of the rock removal mechanism in the new cutting theory

\subsection{Assumptions}

Assumption 1: For simplicity, the two-dimensional orthogonal cutting condition is assumed in this cutting theory. 

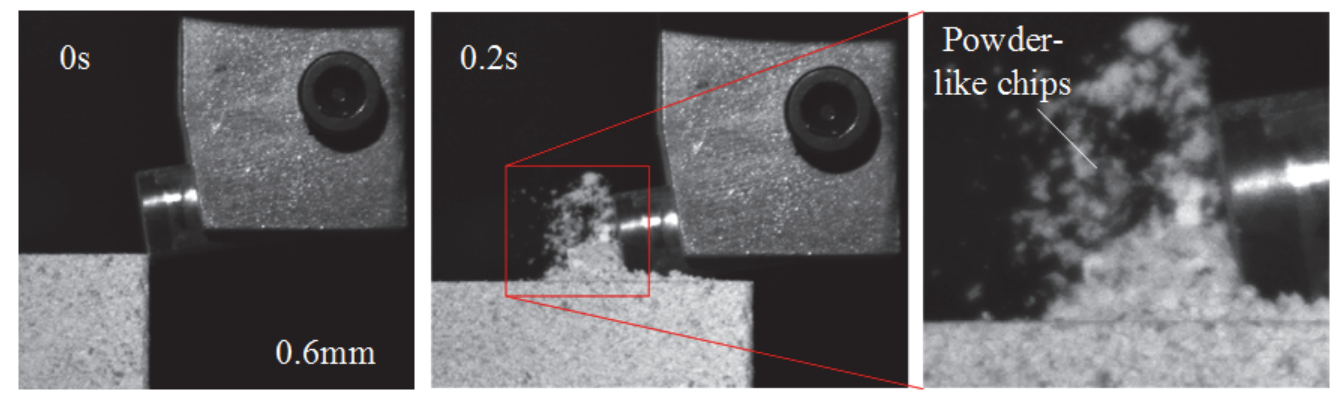

(a)
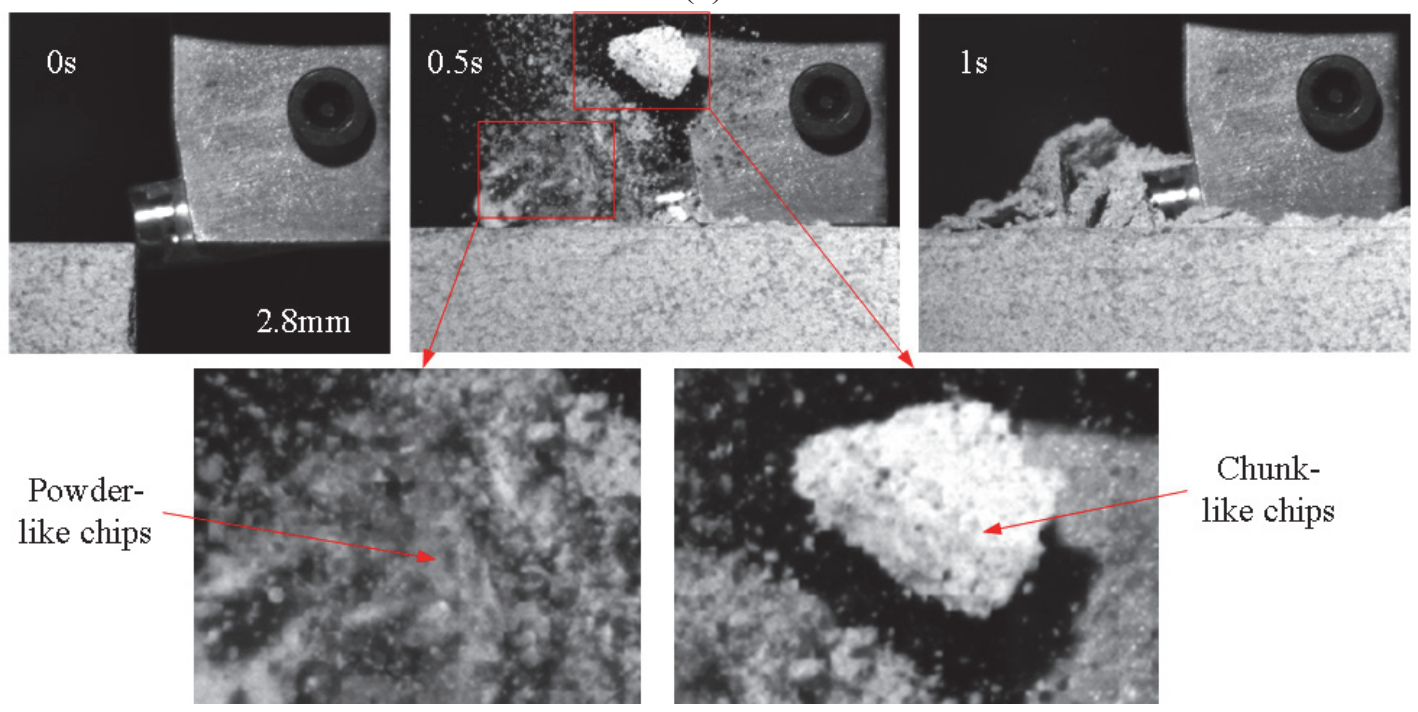

(b)

Fig. 3 Linear cutting of Indiana limestone: a Shallow cut with a depth of cut of $0.6 \mathrm{~mm}$, b Deep cut with a depth of cut of $2.8 \mathrm{~mm}$.

Assumption 2: Figure 3 shows a comparison of the chip formation phenomena between small and large depths of cut in the linear cutting of Indiana limestone. Powdery chips, attributed to the crushing failure mode, exist in both shallow and deep cutting tests but chunk-like chips, attributed to the chipping failure mode, only exist in deep cutting tests. Therefore the theory assumes that chipping and crushing modes simultaneously exist during the cutting process.

Assumption 3: A rock crushing zone, i.e., the red and shaded area in Fig. 2, namely a Tip Crushing Zone (TCZ) is assumed to be formed beneath the cutter's rake face and close to the cutting edge. Since the TCZ is formed due to the crushing of rock, the TCZ boundary is assumed as an arc with a uniform stress distribution equal to the rock's uniaxial compressive strength, $\sigma_{\mathrm{c}}$, from the physical point of view. During the cutting process, the TCZ moves further along the cutting speed direction and continuously forms a stable crushing zone beneath the cutter's rake face.

Assumption 4: The TCZ initiates the chipping of rock. Hence, the crack initiates on the TCZ boundary and propagates following an arc-shaped path towards the free surface of the virgin rock. 
Assumption 5: The chip is assumed to be formed due to tensile stresses along the crack propagation path between the chip and the virgin rock, i.e., the crack is a Mode I crack defined in the theory of fracture mechanics. Since the chip is rapidly formed due to the high speed cutting process, the stress distribution along the arc-shaped crack propagation path right before the separation of the chip from the virgin rock, is assumed to be uniform with a value equal to the rock's tensile stress, $\sigma_{t}$, as shown in Fig. 2. Similar assumption has been made by other researchers $[7,27, \underline{31}, \underline{36}]$.

Assumption 6: The resultant forces exerted from the TCZ and crack propagation path to the chip and their moments are assumed to remain in equilibrium.

Assumption 7: The energy used to form the chip, namely the chipping energy, is assumed to comply with the minimum energy principle proposed in $[17, \underline{32}, \underline{37}$, which is essential to quantitatively determine how the chip forms during the chipping mode.

Assumption 8: The cutting process is assumed to be performed by a sharp cutter, which indicates that all the force components applied by the cutter are from the rake face of the cutter. No friction on the flank face is considered in this theory.

Assumption 9: Due to the brittle failure behavior of the rock cutting processes, it is commonly assumed that the cutting speed has very little influence on the force responses $[\underline{17}, \underline{27}, \underline{38}]$.

\subsection{Cutting Force in a Simple Case}

For simplicity, in this section, the crack was first assumed to initiate at the bottom of the cutting area, which is regarded as a simple model. The crack initiating at arbitrary locations, considered as a general model, will be discussed in next section. As shown in Fig. 4, the crack initiates at Point $C$ which is located at the same horizontal level as the cutter's cutting edge. Since the crack is assumed to be a Mode I crack, i.e., tensile crack due to tensile breakage according to Assumption 5, the crack has to initiate along the direction perpendicular to the maximum principal stress [39], i.e., the horizontal direction.

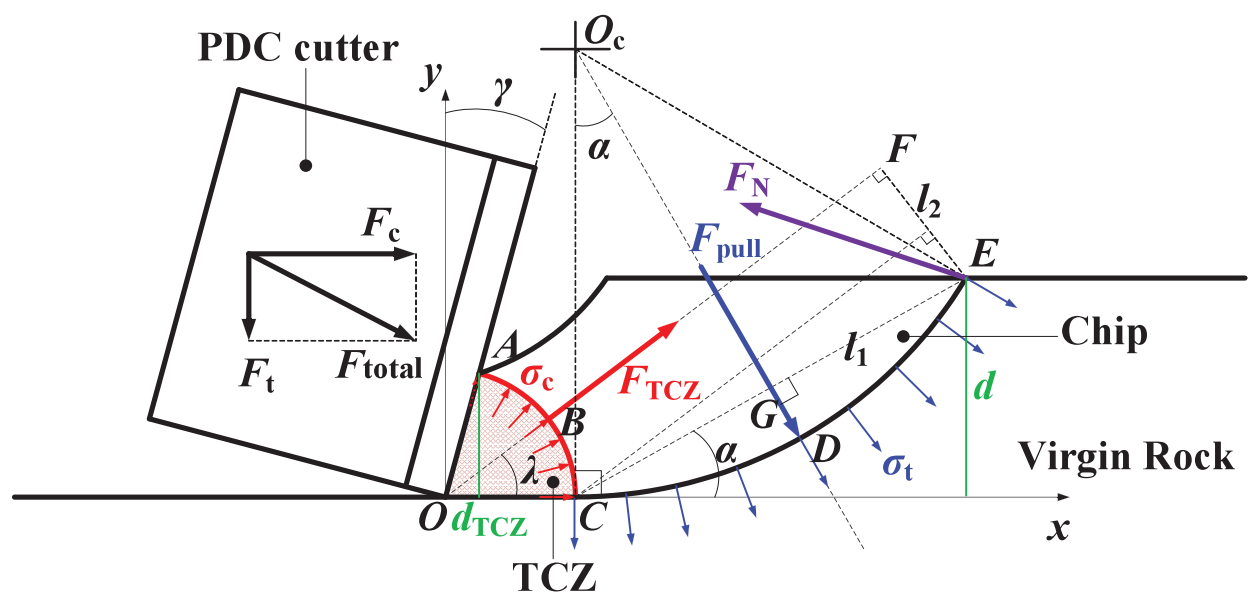

Fig. 4 Schematic of crack initiated along the horizontal direction 
According to Assumption 3, the rock in the TCZ is assumed to be crushed after engaging with the PDC cutter and the stress distribution on the boundary of the TCZ, shown in red, is assumed as a uniform compressive stress with a value equal to the rock's uniaxial compressive strength, $\sigma_{\mathrm{c}}$. Assumption 8 indicates that the cutting and thrust forces are exclusively exerted by the cutter's rake face on the TCZ. Therefore, the horizontal component of the resultant force exerted by the TCZ on the chip, i.e., $F_{\mathrm{TCZ}}$, as shown in Fig. 4, can be considered to be equal in magnitude to the cutting force $F_{\mathrm{c}} . F_{\mathrm{TCZ}}$ has to be determined first in order to derive an expression for $F_{\mathrm{c}}$. Based on the stress distribution on the boundary of the TCZ, $F_{\mathrm{TCZ}}$ can be expressed as:

$$
\overrightarrow{F_{\mathrm{TCZ}}}=w \int_{0}^{2 \lambda} \vec{n} \sigma_{c} r d \theta=w \int_{0}^{2 \lambda}(\cos \theta \vec{i}+\sin \theta \vec{j}) \sigma_{c} r d \theta=w \sigma_{c} r[\vec{i} \sin 2 \lambda-\vec{j}(\cos 2 \lambda-1)]
$$

where $\overrightarrow{F_{\text {TCZ }}}$ is the resultant force exerted at the middle point of the $\operatorname{arc} A B C$, i.e., Point $B$ and pointing upward in the direction normal to $\operatorname{arc} A B C$ at Point $B$. In Eq. (1) $w$ is the cutter's width, $\vec{n}$ is the normal vector on arc $A B C, \lambda$ is the half angle of the TCZ, $r$ is the radius of the TCZ, $\vec{i}$ and $\vec{j}$ are the unit vectors along the horizontal and vertical directions respectively. According to process geometry, the following relations can be given:

$$
\begin{aligned}
& 2 \lambda+\gamma=\frac{\pi}{2} \\
& r=\frac{d_{\mathrm{TCZ}}}{\sin 2 \lambda}
\end{aligned}
$$

where $d_{\mathrm{TCZ}}$ is defined as the depth of the TCZ. Substituting $\lambda$ from Eqs. (2) and $r$ from (3) into Eq. (1) yields:

$$
F_{\mathrm{TCZ}}=2 w \sigma_{c} \frac{d_{\mathrm{TCZ}}}{\cos \gamma} \sin \left(\frac{\pi}{4}-\frac{\gamma}{2}\right)
$$

where $F_{\mathrm{TCZ}}$ is the magnitude of the resultant force. According to Eq. (4), the only unknown parameter, i.e., $d_{\mathrm{TCZ}}$, has to be determined in order to solve for $F_{\mathrm{TCZ}}$. Hence, the ratio of $d_{\mathrm{TCZ}}$ to the depth of cut $d$, i.e., the tip crushing ratio, will be derived based on the equilibrium of moments exerted on the chip. Since $d$ is directly given by the cutting conditions, $d_{\mathrm{TCZ}}$ can then be determined from the tip crushing ratio. To solve the equilibrium of moments, the resultant force exerted by the virgin rock onto the chip, i.e., $F_{\text {pull, }}$, shown in Fig. 4, has to be determined first. According to Assumption 5, the crack propagation path is assumed as an arc, i.e., arc $C D E$, which is tangential to the horizontal direction at Point $C$. Moreover, a uniform tensile stress distribution with the value equal to the rock's tensile strength, $\sigma_{t}$, is assumed to exist along the arc $C D E$ at the moment right before the chip separates from the virgin rock. Therefore, the resultant force exerted by the virgin rock onto the chip can be given by: 
$1^{\text {st }}$ Revision of the Manuscript: Confidential

$$
\overrightarrow{F_{\text {pull }}}=w \int_{0}^{2 \alpha} \vec{N} \cdot \sigma_{t} R d \theta=w \int_{0}^{2 \alpha}(\sin \theta \vec{i}+\cos \theta \vec{j}) \cdot \sigma_{t} R d \theta=w \sigma_{t} R[\vec{j} \cos 2 \alpha-\vec{i}(\cos 2 \alpha-1)]
$$

where $\overrightarrow{F_{\text {pull }}}$ is the resultant force exerted at the middle point of arc $C D E$, i.e., Point $D$ and pointing downward to the direction normal to arc $C D E$ at Point $D, \vec{N}$ is the normal vector at arc $C D E, \alpha$ is the crack inclination angle equal to the half angle of $\operatorname{arc} C D E, R$ is the radius of arc $C D E$. The geometrical relationships in Fig. 4 can be expressed by:

$$
\begin{gathered}
C E=d / \sin \alpha \\
R=0.5 \times C E / \sin \alpha
\end{gathered}
$$

where $\overline{C E}$ is the length of segment $C E$, and $d$ is the depth of cut. Substituting $\overline{C E}$ from Eq. (6) into (7) and then inserting $R$ from Eq. (7) into Eq. (5) yields:

$$
\text { Fpull=otd } / \sin \alpha
$$

Based on Assumption 6, the fores applied on the chip and their moments are assumed to be in equilibrium before the chip separates from the virgin rock. Therefore, a supporting force $F_{\mathrm{N}}$, as shown in Fig. 4 , is defined to equalize the other two forces, i.e., $F_{\text {TCZ }}$ and $F_{\text {pull. }}$. The equilibrium of moments about Point $E$ yields the following condition:

$$
F_{\text {pull }} \times l_{1}-F_{\mathrm{TCZ}} \times l_{2}=0
$$

where $l_{1}$ is the length of segment $E G$ and $l_{2}$ is the length of segment $E F$ which can be expressed by:

$$
\begin{gathered}
l 1=0.5 \times d / \sin \alpha \\
l_{2}=\frac{d}{\sin \alpha} \sin \left(\frac{\pi}{4}-\frac{\gamma}{2}-\alpha\right)+\frac{d_{\mathrm{TCZ}}}{\cos \gamma} \sin \left(\frac{\pi}{4}-\frac{\gamma}{2}\right)
\end{gathered}
$$

Substituting $F_{\text {TCZ }}$ from Eq. (4), $F_{\text {pull }}$ from Eq. (8), $l_{1}$ from Eq. (10), and $l_{2}$ from Eq. (11) into Eq. (9) gives:

$$
\left(\frac{d_{\mathrm{TCZ}}}{d}\right)^{2}+\frac{d_{\mathrm{TCZ}}}{d} \cdot \frac{\cos \gamma \sin \left(\frac{\pi}{4}-\frac{\gamma}{2}-\alpha\right)}{\sin \left(\frac{\pi}{4}-\frac{\gamma}{2}\right) \sin \alpha}-\frac{P \cos ^{2} \gamma}{4 \sin ^{2} \alpha \sin ^{2}\left(\frac{\pi}{4}-\frac{\gamma}{2}\right)}=0
$$

where $d_{\text {TCZ }} / d$ is defined as the tip crushing ratio, while the tensile-compressive ratio, namely $P$, can be defined by:

$$
P=\sigma t / \sigma c
$$


Since $d_{\mathrm{TCZ}} / d$ is always non-negative, from the phyical point of view, the following equation can be drawn:

$$
\frac{d_{\mathrm{TCZ}}}{d}=\frac{-\frac{\cos \gamma \sin \left(\frac{\pi}{4}-\frac{\gamma}{2}-\alpha\right)}{\sin \left(\frac{\pi}{4}-\frac{\gamma}{2}\right) \sin \alpha}+\sqrt{\Delta}}{2}
$$

where

$$
\Delta=\frac{\cos ^{2} \gamma\left[\sin ^{2}\left(\frac{\pi}{4}-\frac{\gamma}{2}-\alpha\right)+P\right]}{\sin ^{2} \alpha \sin ^{2}\left(\frac{\pi}{4}-\frac{\gamma}{2}\right)}
$$

Substitution of $\Delta$ from Eq. (15) into Eq. (14) yields:

$$
\frac{d_{\mathrm{TCZ}}}{d}=\frac{\cos \gamma}{2 \sin \left(\frac{\pi}{4}-\frac{\gamma}{2}\right)} F
$$

where

$$
\begin{aligned}
F & =-\frac{\sin (\varepsilon-\alpha)}{\sin \alpha}+\sqrt{\frac{\sin ^{2}(\varepsilon-\alpha)}{\sin ^{2} \alpha}+P \csc ^{2} \alpha} \\
& =\cos \varepsilon-\sin \varepsilon \cot \alpha+\sqrt{\left(\sin ^{2} \varepsilon+P\right) \cot ^{2} \alpha-\sin 2 \varepsilon \cot \alpha+P+\cos ^{2} \varepsilon}
\end{aligned}
$$

and where

$$
\varepsilon=\frac{\pi}{4}-\frac{\gamma}{2}
$$

As it can be seen, to evaluate the tip crushing ratio, given by Eq. (16), with reference to Eqs. (17) and (18), the rake angle $\gamma$, the tensile-compressive ratio $P$, and the crack inclination angle $\alpha$ need to be known. The first two are known since they are defined by the given cutting conditions, while $\alpha$ needs to be determined. Its determination is addressed next.

Based on the minimum energy principle, mentioned under Assumption 7, the crack inclination angle $\alpha$ can be determined by minimizing the chipping energy, $E_{\mathrm{c}}$, i.e.: 


$$
\frac{\partial}{\partial \alpha}\left(E_{c}\right)=0
$$

Where

$$
E_{\mathrm{c}}=F_{\mathrm{c}} \times L
$$

and where $L$ is the total distance that the cutter has moved. According to Eq. (1), the cutting force used to chip the rock can be determined by:

$$
F_{\mathrm{c}}=\vec{i} \cdot \overrightarrow{F_{\mathrm{TCZ}}}=\sigma_{c} w r \sin 2 \lambda=\sigma_{c}\left(\frac{d_{\mathrm{TCZ}}}{d}\right) w d
$$

Therefore, substituting $F_{\mathrm{c}}$ from Eq. (21) into Eq. (20) and then inserting $E_{\mathrm{c}}$ from Eq. (20) into Eq. (19) yields:

$$
\frac{\partial}{\partial \alpha}\left(\frac{d_{\mathrm{TCZ}}}{d}\right)=0
$$

According to Eqs. (16), (17) and (22), $\frac{\partial F}{\partial \alpha}$ has to be zero and can be given by the following expression:

$$
\begin{aligned}
& \frac{\partial F}{\partial \alpha}=\sin \varepsilon \csc ^{2} \alpha \\
& -\frac{2\left(\sin ^{2} \varepsilon+P\right) \cot \alpha-\sin 2 \varepsilon}{2 \sqrt{\left(\sin ^{2} \varepsilon+P\right) \cot ^{2} \alpha-\sin 2 \varepsilon \cot \alpha+P+\cos ^{2} \varepsilon}} \csc ^{2} \alpha=0
\end{aligned}
$$

Simplification of Eq. (23) yields:

$$
4 P\left(P+\sin ^{2} \varepsilon\right) \cot ^{2} \alpha-4 P \sin 2 \varepsilon \cot \alpha-4 P \sin ^{2} \varepsilon=0
$$

Since $\alpha$ is assumed to be positive, $\alpha$ can be solved from Eq. (24), i.e.:

$$
\alpha=\arctan \frac{2\left(P+\sin ^{2} \varepsilon\right)}{\sin 2 \varepsilon+\sqrt{\sin ^{2} 2 \varepsilon+4\left(P+\sin ^{2} \varepsilon\right) \sin ^{2} \varepsilon}}
$$

Substitution of $P$ from Eq. (13) and $\varepsilon$ from Eq. (18) into Eq. (25) yields: 


$$
\alpha=\arctan \frac{2\left(\frac{\sigma_{t}}{\sigma_{c}}+\sin ^{2}\left(\frac{\pi}{4}-\frac{\gamma}{2}\right)\right)}{\cos \gamma+\sqrt{\cos ^{2} \gamma+4 \frac{\sigma_{t}}{\sigma_{c}} \sin ^{2}\left(\frac{\pi}{4}-\frac{\gamma}{2}\right)+4 \sin ^{4}\left(\frac{\pi}{4}-\frac{\gamma}{2}\right)}}
$$

Therefore, substituting $\alpha$ from Eq. (26) and $F$ from Eq. (17) into Eq. (16) gives the closed form expression of the tip crushing ratio, i.e.:

$$
\begin{aligned}
& \frac{d_{\mathrm{TCZ}}}{d}=\cos \gamma\left[-\frac{\cos \gamma}{4 \sin ^{2}\left(\frac{\pi}{4}-\frac{\gamma}{2}\right)+4 \frac{\sigma_{t}}{\sigma_{c}}}-\frac{\sqrt{\cos ^{2} \gamma+4 \frac{\sigma_{t}}{\sigma_{c}} \sin ^{2}\left(\frac{\pi}{4}-\frac{\gamma}{2}\right)+4 \sin ^{4}\left(\frac{\pi}{4}-\frac{\gamma}{2}\right)}}{4 \sin ^{2}\left(\frac{\pi}{4}-\frac{\gamma}{2}\right)+4 \frac{\sigma_{t}}{\sigma_{c}}}\right. \\
& \left.+\frac{\sqrt{\frac{\sigma_{t}}{\sigma_{c}}+1}}{2 \sin \left(\frac{\pi}{4}-\frac{\gamma}{2}\right)}+\frac{1}{2} \cot \left(\frac{\pi}{4}-\frac{\gamma}{2}\right)\right]
\end{aligned}
$$

Finally, substituting $d_{\mathrm{TCZ}} / d$ from Eq. (27) into Eq. (21) yields the close form of the cutting force, i.e.:

$$
\begin{aligned}
& F_{\mathrm{c}}=w \sigma_{c} d \cos \gamma\left[-\frac{\cos \gamma}{4 \sin ^{2}\left(\frac{\pi}{4}-\frac{\gamma}{2}\right)+4 \frac{\sigma_{t}}{\sigma_{c}}}\right. \\
& \left.-\frac{\sqrt{\cos ^{2} \gamma+4 \frac{\sigma_{t}}{\sigma_{c}} \sin ^{2}\left(\frac{\pi}{4}-\frac{\gamma}{2}\right)+4 \sin ^{4}\left(\frac{\pi}{4}-\frac{\gamma}{2}\right)}}{4 \sin ^{2}\left(\frac{\pi}{4}-\frac{\gamma}{2}\right)+4 \frac{\sigma_{t}}{\sigma_{c}}}+\frac{\sqrt{\frac{\sigma_{t}}{\sigma_{c}}+1}}{2 \sin \left(\frac{\pi}{4}-\frac{\gamma}{2}\right)}+\frac{1}{2} \cot \left(\frac{\pi}{4}-\frac{\gamma}{2}\right)\right]
\end{aligned}
$$

At this stage, the cutting force required to form a horizontal crack can be explicitly determined by Eq. (28) with the knowledge of the corresponding parameters. The required parameters include process parameters, i.e., cutter's width $w$, depth of cut $d$, and rake angle $\gamma$, material parameters, i.e., the rock's uniaxial compressive strength $\sigma_{\mathrm{c}}$ and its tensile strength $\sigma_{\mathrm{t}}$.

In reality, however, the crack usually initiates at a random location and propagates along an arbitrary direction rather than the horizontal direction. Therefore, a more general cutting force model has to be derived. This follows in the next section.

\subsection{A General Cutting Force Model}

In reality, the crack does not necessarily initiate horizontally as it was assumed for simplicity in Section 2.3. Therefore, a more general cutting force model has to be formulated. As shown in Fig. 5, the crack is assumed to initiate at an arbitrary location $C$ on the boundary of the TCZ. The crack propagation path is perpendicular to the boundary of TCZ at point $C$. In this way, the chip will be formed above line $C K$ 
following a similar mechanism as for the simple model discussed in Section 2.2, while the rest of the rock below line $C K$ will fail in a pure crushing mode. Hence, both chipping and crushing mechanisms are naturally included in this general force model. The total cutting force can be expressed by:

$$
F_{\mathrm{c}}=\vec{i} \cdot\left(\overrightarrow{F_{\mathrm{TCZ}-\mathrm{c}}}+\overrightarrow{F_{\mathrm{TCZ}-\mathrm{cr}}}\right)=\vec{i} \cdot w \int_{0}^{\frac{\pi}{2}-\gamma} \vec{n} \cdot \sigma_{c} \frac{D_{\mathrm{TCZ}}}{\cos \gamma} d \theta=w \sigma_{c} D_{\mathrm{TCZ}}
$$

where $\overrightarrow{F_{\text {TCZ-c }}}$ and $\overrightarrow{F_{\text {TCZ-cr }}}$ are the resultant chipping and crushing forces respectively, $w$ is the cutter's width, $D_{\mathrm{TCZ}}$ is the depth of the TCZ for the general model, $\vec{n}$ is the normal vector along the TCZ boundary, and $\vec{i}$ is the unit vector along the horizontal direction. According to Eq. (29), $D_{\mathrm{TCZ}}$ has to be determined to fully predict $F_{\mathrm{c}}$. Therefore, in a manner similar to the simpler model in Section 2.2, the tip crushing ratio, i.e., $D_{\text {TCZ }} / D$, has to be determined.

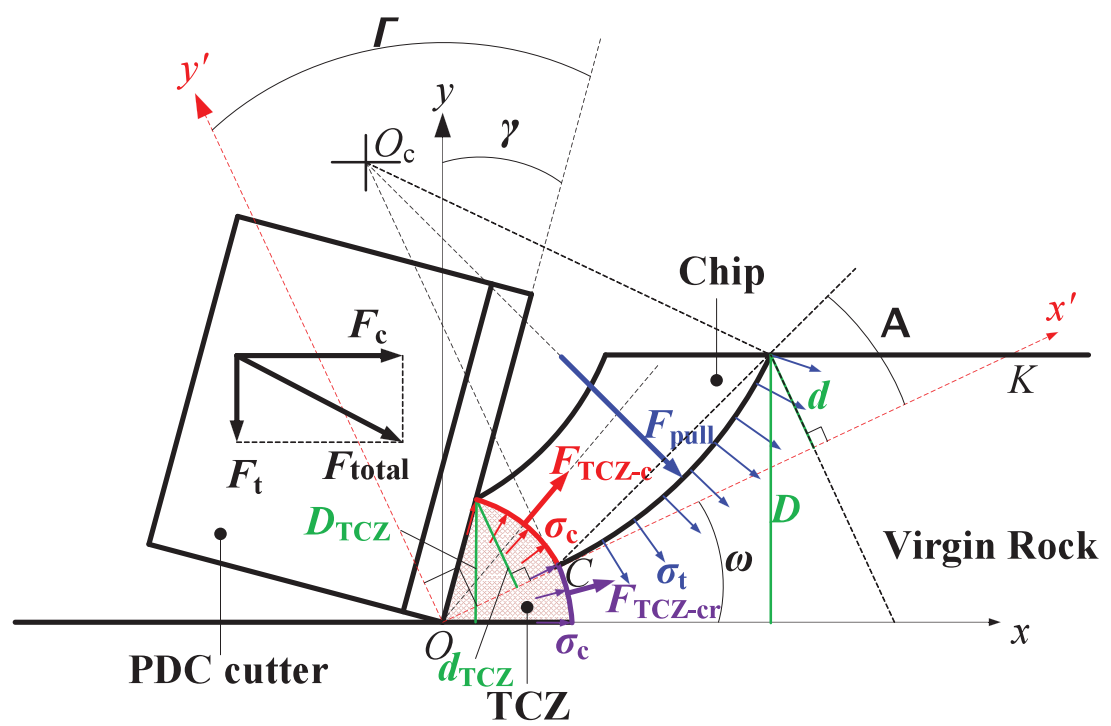

Fig. 5 Schematic of the rock removal mechanism in the arbitrary chipping mode

From the physical point of view, line OCK, shown in Fig. 5, divides the rock into two portions, i.e., the upper portion affected by the chipping action and the lower portion affected by the crushing action. Therefore, the upper portion is defined as the chipping affected zone, while the lower portion is defined as the crushing affected zone. The angle $\omega$, namely the chipping-crushing transition angle, is defined to characterize the transition boundary between these two affected zones.

Similarities in the chipping action exist between the simple and the general models described in Sections 2.2 and 2.3 respectively. As shown in Fig. 4, the chipping action occurs beneath the cutter's rake face but above the $\mathrm{x}$-axis, i.e., line $O C x$ which can be defined as the chipping affected zone in the simple model. Similarly, the chipping affected zone in the general model, shown in Fig. 5, is the rock beneath the cutter's rake face but above the $x$ '-axis, i.e., line $O C K$. Therefore, by rotating the chipping affected zone in the simple model, shown in Fig. 4 , by the angle $\omega$, the chipping affected zone in the general model can 
be derived as shown in Fig. 5. In other words, as shown in Fig. 5, the coordinate system x'Oy' is defined by rotating the original coordinate system $x O y$ by $\omega$. Therefore, the chipping action for the general model with an equivalent rake angle $\Gamma$, equivalent depth of cut $d$, and equivalent depth of the TCZ $d_{\mathrm{TCZ}}$, as observed in the coordinate system $x^{\prime} O y^{\prime}$, as shown in Fig. 5, is completely equivalent to that in the simple model with a rake angle $\gamma$, depth of cut $d$, and depth of the TCZ $d_{\mathrm{TCZ}}$ observed in the original coordinate system $x O y$ shown in Fig. 4.

Therefore, based on the above-stated similarity, the ratio of $d_{\mathrm{TCZ}}$ to $d$ in the general model can be derived by substituting the equivalent rake angle $\Gamma$ into Eq. (27), i.e.:

$$
\begin{aligned}
& m=\frac{d_{\mathrm{TCZ}}}{d}=\cos \Gamma \times\left[-\frac{\cos \Gamma}{4 \sin ^{2}\left(\frac{\pi}{4}-\frac{\Gamma}{2}\right)+4 \frac{\sigma_{t}}{\sigma_{c}}}-\frac{\sqrt{\cos ^{2} \Gamma+4 \frac{\sigma_{t}}{\sigma_{c}} \sin ^{2}\left(\frac{\pi}{4}-\frac{\Gamma}{2}\right)+4 \sin ^{4}\left(\frac{\pi}{4}-\frac{\Gamma}{2}\right)}}{4 \sin ^{2}\left(\frac{\pi}{4}-\frac{\Gamma}{2}\right)+4 \frac{\sigma_{t}}{\sigma_{c}}}\right. \\
& \left.+\frac{\sqrt{\frac{\sigma_{t}}{\sigma_{c}}+1}}{2 \sin \left(\frac{\pi}{4}-\frac{\Gamma}{2}\right)}+\frac{1}{2} \cot \left(\frac{\pi}{4}-\frac{\Gamma}{2}\right)\right]
\end{aligned}
$$

Since the coordinate system $x^{\prime} O y^{\prime}$ is derived by rotating the coordinate system $x O y$ by $\omega$, the relationship between $\Gamma$ and $\gamma$ is given by:

$$
\Gamma=\gamma+\omega
$$

According to the geometrical relationships shown in Fig. 5, the depth of cut $D$ and depth of the TCZ $D_{\text {TCZ }}$ can be expressed by:

$$
\begin{gathered}
D=\left[\left(\frac{d_{\mathrm{TCZ}}}{\cos (\gamma+\omega)}+\frac{d}{\tan \mathrm{A}}\right) \tan \omega+d\right] \cos \omega \\
\frac{D_{\mathrm{TCZ}}}{\sin \left(\frac{\pi}{2}-\gamma\right)}=\frac{d_{\mathrm{TCZ}}}{\sin \left(\frac{\pi}{2}-\gamma-\omega\right)}
\end{gathered}
$$

Combining Eqs. (30), (31), (32) and (33) to derive the relationship between $D_{\mathrm{TCZ}}$ and $D$, one obtains:

$$
M=\frac{D_{\mathrm{TCZ}}}{D}=\frac{\sin \left(\frac{\pi}{2}-\gamma\right)}{\sin \left(\frac{\pi}{2}-\gamma-\omega\right)} \times \frac{m}{\frac{m \sin \omega}{\cos (\gamma+\omega)}+\frac{\sin \omega}{\tan \mathrm{A}}+\cos \omega}
$$


where $M$ is the tip crushing ratio in the general model, and $\mathrm{A}$ is the equivalent crack inclination angle. According to Eq. (26), A can be expressed as:

$$
\mathrm{A}=\arctan \frac{2\left(\frac{\sigma_{t}}{\sigma_{c}}+\sin ^{2}\left(\frac{\pi}{4}-\frac{\Gamma}{2}\right)\right)}{\cos \Gamma+\sqrt{\cos ^{2} \Gamma+4 \frac{\sigma_{t}}{\sigma_{c}} \sin ^{2}\left(\frac{\pi}{4}-\frac{\Gamma}{2}\right)+4 \sin ^{4}\left(\frac{\pi}{4}-\frac{\Gamma}{2}\right)}}
$$

Combining Eqs. (29), (31), (34) and (35), the cutting force for the general model can be expressed by:

$$
\begin{aligned}
& F_{\mathrm{c}}=w \sigma_{c} D \frac{\sin \left(\frac{\pi}{2}-\gamma\right)}{\sin \left(\frac{\pi}{2}-\gamma-\omega\right)} \times \\
& \frac{m \sin \omega}{\cos (\gamma+\omega)}+\sin \omega \frac{\cos (\gamma+\omega)+\sqrt{\cos ^{2}(\gamma+\omega)+4 \frac{\sigma_{t}}{\sigma_{c}} \sin ^{2}\left(\frac{\pi}{4}-\frac{\gamma+\omega}{2}\right)+4 \sin ^{4}\left(\frac{\pi}{4}-\frac{\gamma+\omega}{2}\right)}}{2\left(\frac{\sigma_{t}}{\sigma_{c}}+\sin ^{2}\left(\frac{\pi}{4}-\frac{\gamma+\omega}{2}\right)\right)}+\cos \omega
\end{aligned}
$$

where

$$
\begin{aligned}
& m=\cos (\gamma+\omega) \times\left[-\frac{\cos (\gamma+\omega)}{4 \sin ^{2}\left(\frac{\pi}{4}-\frac{\gamma+\omega}{2}\right)+4 \frac{\sigma_{t}}{\sigma_{c}}}\right. \\
& -\frac{\sqrt{\cos ^{2}(\gamma+\omega)+4 \frac{\sigma_{t}}{\sigma_{c}} \sin ^{2}\left(\frac{\pi}{4}-\frac{\gamma+\omega}{2}\right)+4 \sin ^{4}\left(\frac{\pi}{4}-\frac{\gamma+\omega}{2}\right)}}{4 \sin ^{2}\left(\frac{\pi}{4}-\frac{\gamma+\omega}{2}\right)+4 \frac{\sigma_{t}}{\sigma_{c}}} \\
& \left.+\frac{\sqrt{\frac{\sigma_{t}}{\sigma_{c}}+1}}{2 \sin \left(\frac{\pi}{4}-\frac{\gamma+\omega}{2}\right)}+\frac{1}{2} \cot \left(\frac{\pi}{4}-\frac{\gamma+\omega}{2}\right)\right]
\end{aligned}
$$

In this way, the cutting force can be derived, based on Eq. (36), for the general model in which the crack initiates at an arbitrary location on the TCZ boundary. All the parameters required in Eqs. (36) and (37) for evaluating the cutting force are directly available except for the newly defined parameter $\omega$ which needs to be calibrated. According to definition, the increase of $\omega$ will decrease the size of the chipping affected zone as shown in Fig. 5 and, in turn, decrease the size of the formed chips. Even though the physical meaning of $\omega$ is intuitive, the accurate value of $\omega$ is unknown without experimental calibration. In this work, $\omega$ has been found to change linearly with respect to depth of cut at a constant rake angle, 
which can be calibrated by using linear regression techniques. The calibration of $\omega$ will be presented in detail in Section 4.

\subsection{Relationship between Cutting and Thrust Forces}

One of the two orthogonal components of the resultant force, i.e., the cutting force, has been derived in Section 2.3. In this section, the other component, i.e., the thrust force will be related to the cutting force in accordance with the physical interaction between the cutter and rock on the cutter's rake face.

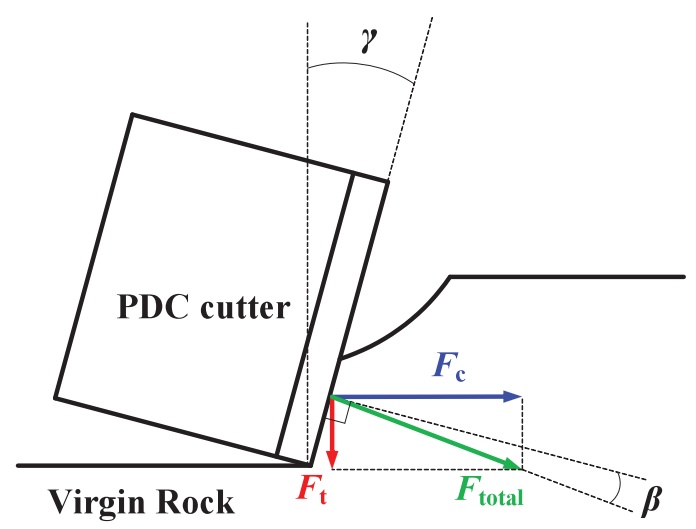

Fig. 6 Relationship between the cutting and thrust forces

Figure 6 shows the decomposition of the resultant force $F_{\text {total }}$ exerted on the rake face of the PDC cutter into two orthogonal force components, i.e., cutting and thrust forces which can be related by:

$$
\frac{F_{t}}{F_{c}}=\tan (\gamma+\beta)
$$

where $\beta$ is the mean friction angle between the rock and the cutter's rake face. $\beta$ characterizes the frictional interaction between the cutter's rake face and the rock from an average point of view. The larger $\beta$ is, the more likely the chip will stick to the rake face.

Replacing $F_{\mathrm{c}}$ in Eq. (38) with Eq. (36) yields:

$$
F_{t}=\tan (\gamma+\beta) w \sigma_{c} D \frac{\sin \left(\frac{\pi}{2}-\gamma\right)}{\sin \left(\frac{\pi}{2}-\gamma-\omega\right)} \times
$$$$
\frac{m \sin \omega}{\cos (\gamma+\omega)}+\sin \omega \frac{\cos (\gamma+\omega)+\sqrt{\cos ^{2}(\gamma+\omega)+4 \frac{\sigma_{t}}{\sigma_{c}} \sin ^{2}\left(\frac{\pi}{4}-\frac{\gamma+\omega}{2}\right)+4 \sin ^{4}\left(\frac{\pi}{4}-\frac{\gamma+\omega}{2}\right)}}{2\left(\frac{\sigma_{t}}{\sigma_{c}}+\sin ^{2}\left(\frac{\pi}{4}-\frac{\gamma+\omega}{2}\right)\right)}+\cos \omega
$$ 
As it has been shown, both the cutting and thrust forces can be determined based on Eqs. (36) and (39) respectively. It should be noted that all the parameters involved in the closed form expressions of the cutting and thrust forces, i.e., Eq. (36) and (39) are automatically determined as long as the cutting conditions are specified, except for two parameters, i.e., $\omega$ and $\beta$. According to Eqs. (36) and (39), $\omega$ has to be determined to evaluate the value of the cutting force, while $\beta$ has to be determined to derive the thrust force as a function of the cutting force. As mentioned in Section 2.3, $\omega$ is linearly related to the depth of cut for a constant rake angle. Moreover, as it will be shown, $\beta$ can be linearly related to the rake angle and independent of the depth of cut. Therefore, given the available force data from preliminary tests, the linear change of $\omega$ with respect to the depth of cut and the linear trend of $\beta$ with respect to the rake angle can be accurately calibrated using linear regression techniques. In this way, both $\omega$ and $\beta$ can be determined for different rake angles and depths of cut for future predictions. The detailed calibration procedures will be presented in Section 4.

\section{Experiments}

This section will briefly introduce the linear rock cutting facility and testing procedures, followed by the experimental results which will be used to evaluate the above-derived cutting theory in the next section.

\subsection{Experimental Setup}

A linear rock cutting testbed has been developed at Northwestern University. As shown in Fig. 7, the system includes a XYZ moving stage (with a maximal moving speed of $170 \mathrm{~mm} / \mathrm{s}$ ), a tool holder with multiple rake angle adjustment inserts, a 3-component Kistler dynamometer (Kistler 9255A), three PCB unidirectional accelerometers (PCB 308B15), and a National Instruments data acquisition system (NI PXI-1036 chassis with NI PXI 6123 card). More detailed information can be found in the authors' previous paper [40-42].

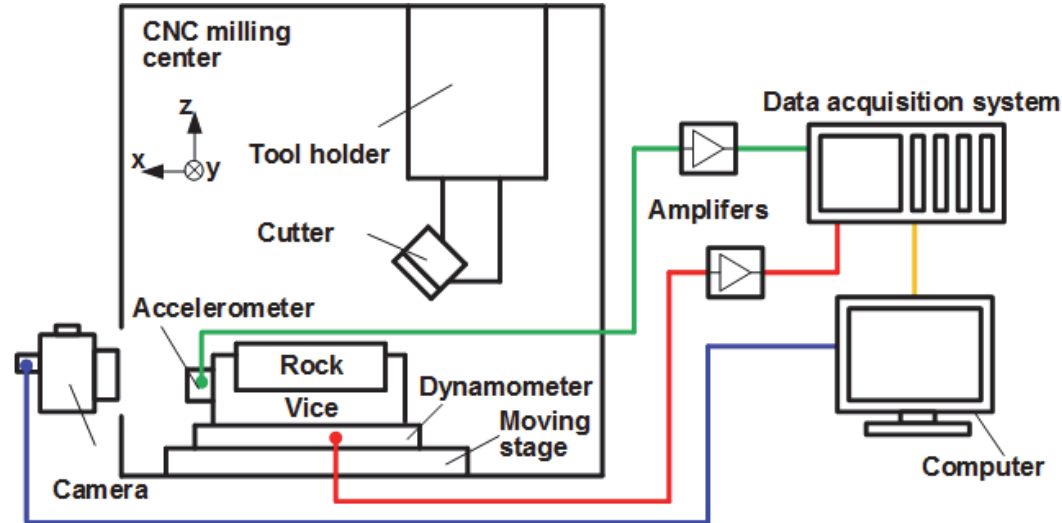

(a)

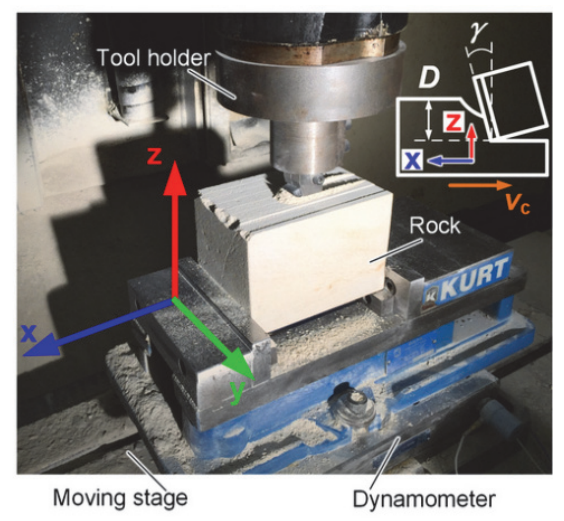

(b)

Fig. 7 Configuration of the linear rock cutting testbed: (a) Testbed schematic; (b) Photograph of the rockcutter interface inside the testbed.

In this work, the selected rock sample is Indiana limestone, as shown in Fig. 8, with an uniaxial compressive and tensile strengths of 34.5MPa [43] and 3.45MPa [44] respectively. The cutting speed was set to a constant value of $63.5 \mathrm{~mm} / \mathrm{s}$. The PDC cutter's width $w$ is approximately $19 \mathrm{~mm}$ as shown in Fig. 9. The cutting edge of the cutter is approximately straight while the two side edges of the cutter are 
curved to avoid stress concentrations on the both ends of the cutting edge. The PDC cutter is considered to be sharp for all the cutting tests conducted in this work.

At the beginning of each test, the cutter's rake angle is adjusted to a specific value by the rake angle adjustment inserts while the depth of cut is adjusted to a certain depth by the moving stage. The experiments include 3 levels of rake angles, i.e., $10^{\circ}, 20^{\circ}$, and $30^{\circ}$ and 13 levels of depths of cut, i.e., 0.2 , $0.4,0.6,0.8,1,1.2,1.4,1.6,1.8,2,2.4,2.8$ and $3 \mathrm{~mm}$. For each combination of rake angle and depth of cut, three repeated tests were conducted to minimize experimental errors. During each test, the moving stage moves the rock against the PDC cutter to achieve a linear motion between the rock and the cutter. Since the selected depths of cut are much smaller than the cutter's width, the cutting process in this work can be assumed to remain two-dimensional orthogonal cutting.

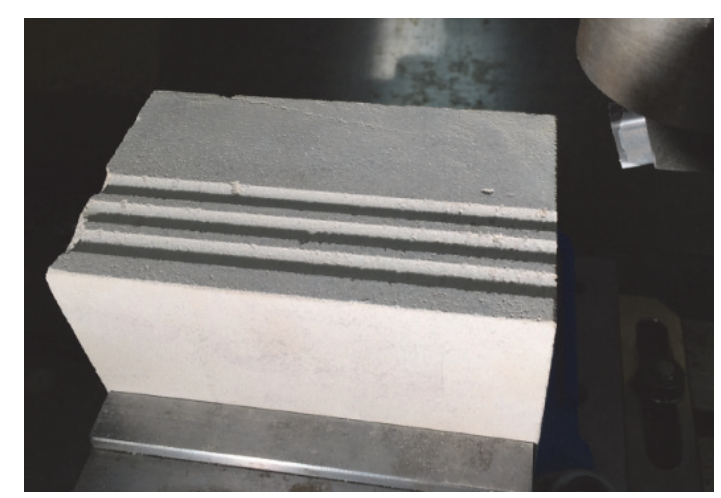

Fig. 8 Indiana limestone sample after three linear cuts

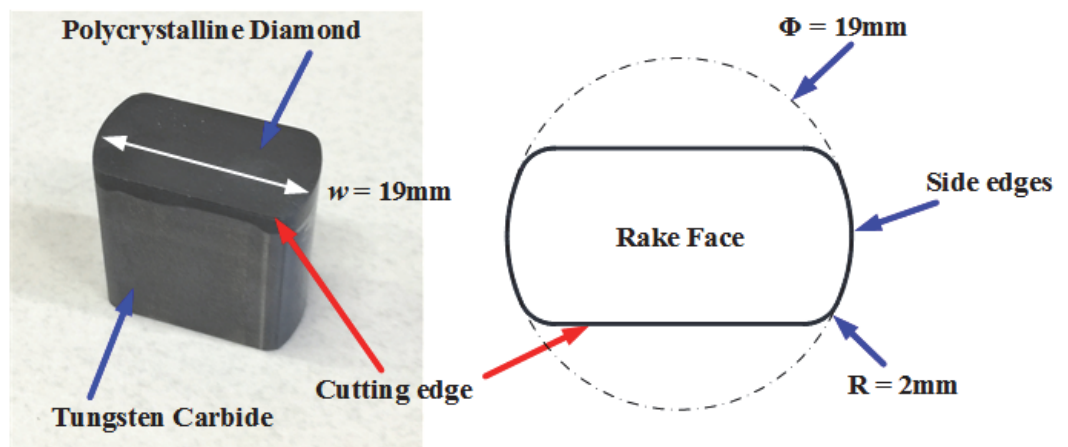

Fig. 9 PDC cutter with custom-designed geometry

\subsection{Results}

The force responses were captured by the above-mentioned 3-component dynamometer with a $20 \mathrm{kHz}$ sampling rate and filtered by a low-pass digital filter with a cut-off frequency of $300 \mathrm{~Hz}$. Figure 10 shows the force responses for a cutting test with a $20^{\circ}$ rake angle and a $1.6 \mathrm{~mm}$ depth of cut. The forces are observed to oscillate during the cutting process due to the continuous crushing and chipping processes. For all the tests, the force responses were averaged within a specific sampling period of $1 \mathrm{~s}$. The moment that the sampling period starts is $0.3 \mathrm{~s}$ after the moment when the tool started to engage the rock. In this way, three average force components, i.e., average cutting, thrust and side forces can be derived for each test. The average cutting force is pointing along the cutting speed direction, i.e., x-direction as illustrated in Fig. 7(b) while the average thrust force is vertical to the top surface of the rock, i.e., z-direction as 
illustrated in Fig. 7(b). The average side force is orthogonal to both the average cutting and thrust forces. The process parameters and corresponding force responses are listed in Table A.1 in the Appendix. As shown in Table A.1, the average cutting and thrust forces increase as the depth of cut and rake angle increase, while the average side force remains a very small value satisfying the assumption on orthogonal cutting.

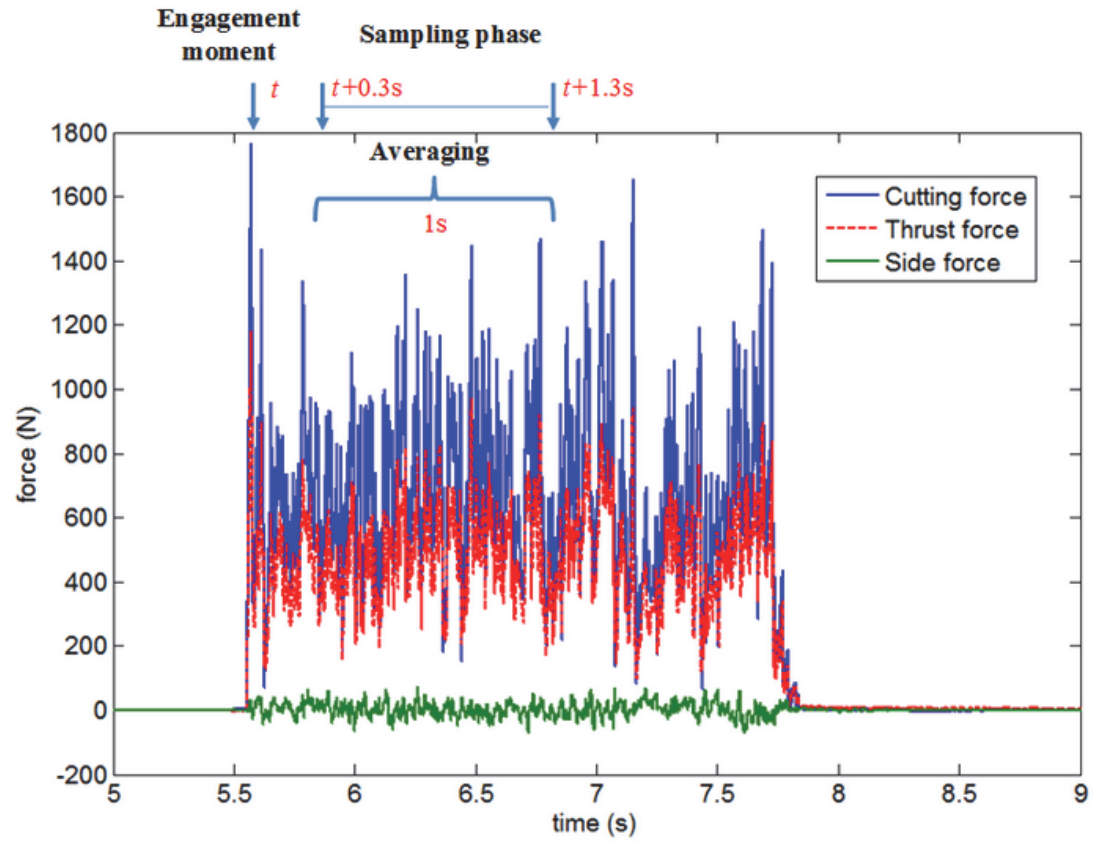

Fig. 10 Force responses from one of the linear cutting tests in this work

\section{Model Evaluations}

This section will utilize the experimental data to calibrate the two parameters defined in the new force model, i.e., the chipping-crushing transition angle $\omega$ and the mean friction angle $\beta$. The calibrated $\omega$ and $\beta$ will then be adopted to predict the cutting and thrust force responses, as discussed in Section 2.4.

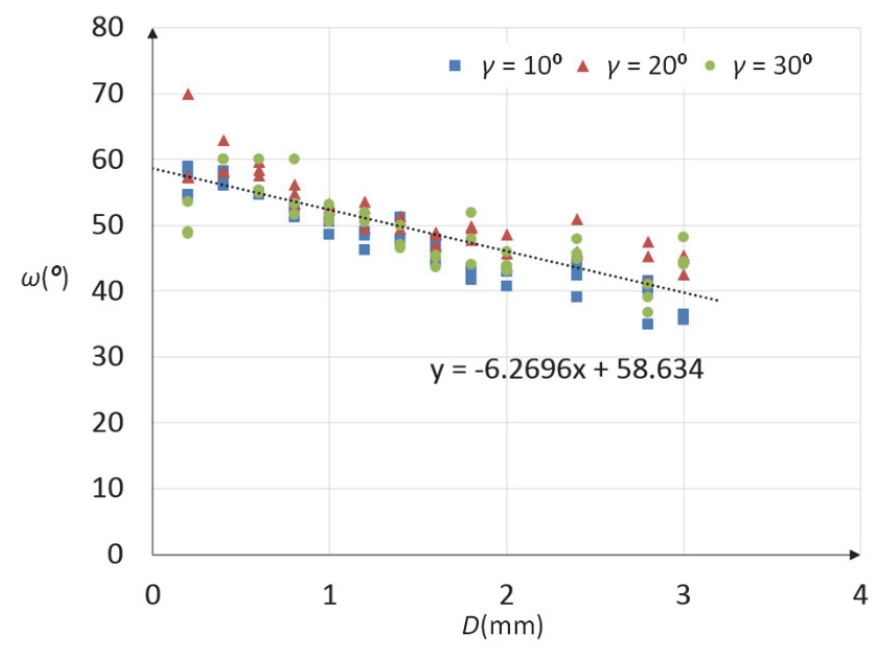

Fig. 11 Linear trends of the chipping-crushing transition angle with respect to depth of cut 


\subsection{Calibration of the Chipping-Crushing Transition Angle}

According to Eqs. (36) and (37), for each test listed in Table A.1, all the parameters except for $\omega$ are given/known based on the specific cutting conditions. Moreover, the average cutting force has also been experimentally derived. Therefore, $\omega$ is the only unknown parameter in Eq. (36) and can be determined numerically.

As shown in Fig. 11, $\omega$ is decreasing linearly with the increase of the depth of cut $D$. Therefore, $\omega$ was be calibrated using the linear regression method to yield:

$$
\omega\left({ }^{\circ}\right)=-6.2696 D(\mathrm{~mm})+58.634
$$

\subsection{Calibration of the Mean Friction Angle between the Rock and the Cutter's Rake Face}

Figure 12 shows the linear increase of the thrust force as the cutting force increases at a constant rake angle $\gamma$. According to Eq. (38), $\beta$ can be solved by:

$$
\beta=\arctan \left(\frac{F_{t}}{F_{c}}\right)-\gamma
$$

where the ratios of the thrust to cutting forces can be derived based on the slopes of the three lines plotted in Fig. 12.The ratio of thrust to cutting forces remains constant at each level of the rake angle, as shown in Fig. 13. Hence $\beta$ can be linearly related to $\gamma$ by:

$$
\beta\left(^{\circ}\right)=-0.5742 \gamma\left(\left(^{\circ}\right)+25.782\right.
$$

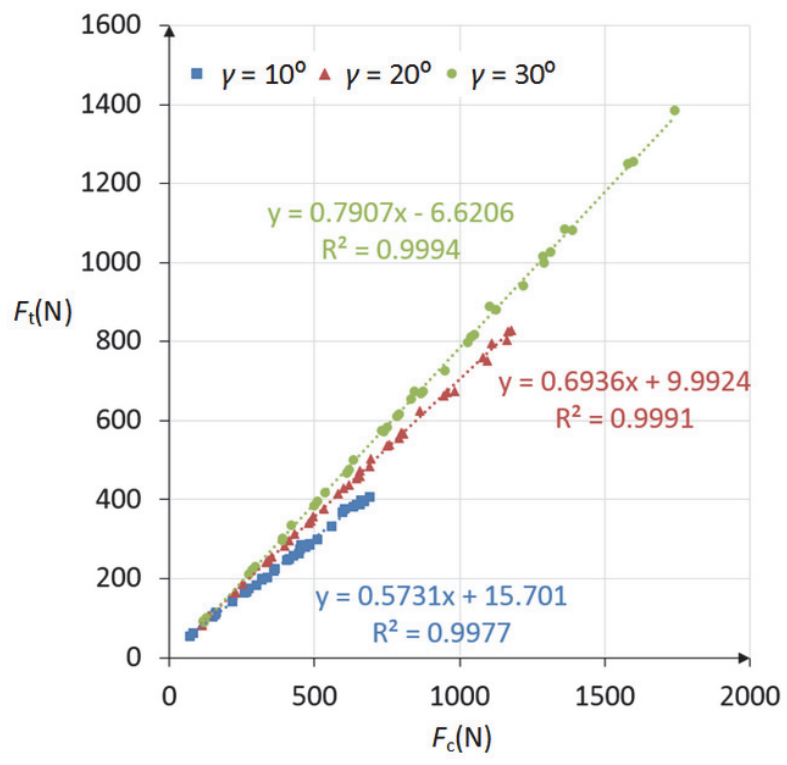

Fig. 12 Relationship between the cutting and thrust forces with 3 levels of rake angles 


\subsection{Force predictions}

As discussed above, given the depth of cut $D$ and rake angle $\gamma, \omega$ and $\beta$ can be determined by Eqs. (40) and (42). Substituting the calibrated values of $\omega$ and $\beta$ and the pre-defined process parameters $w, D, \gamma, \sigma_{\mathrm{t}}$, and $\sigma_{\mathrm{c}}$ into Eqs. (36), (37) and (39) will give the predictions of the desired cutting and thrust forces. The predicted values and their prediction errors are listed in Table A.1. The average prediction errors of the cutting and thrust forces for all the data points in Table A.1 are about 6\% and 8\% respectively. Figure 14 compares the experimental results with the predictions. In this way, the non-linear force responses can be simply predicted by calibrating two parameters $\omega$ and $\beta$ using the above-described linear regression method.

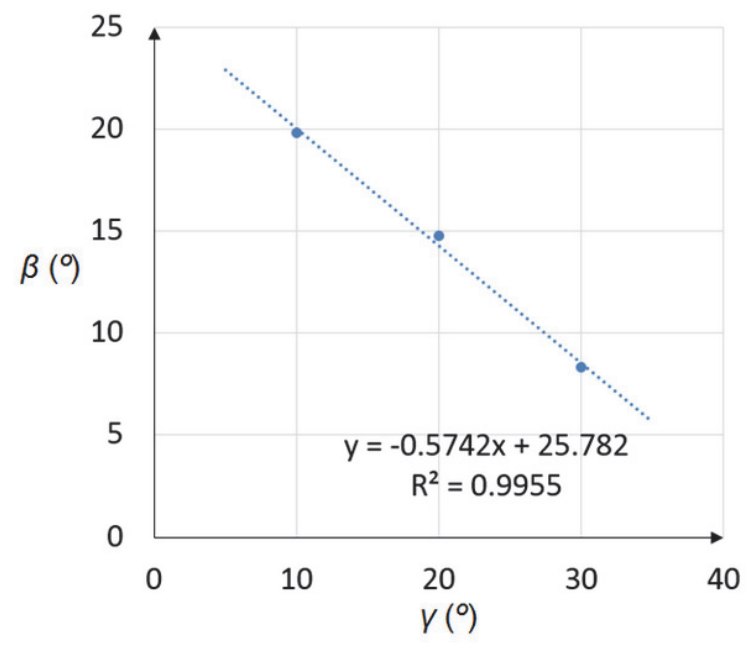

Fig. 13 Linear relationship between the rake and mean friction angles
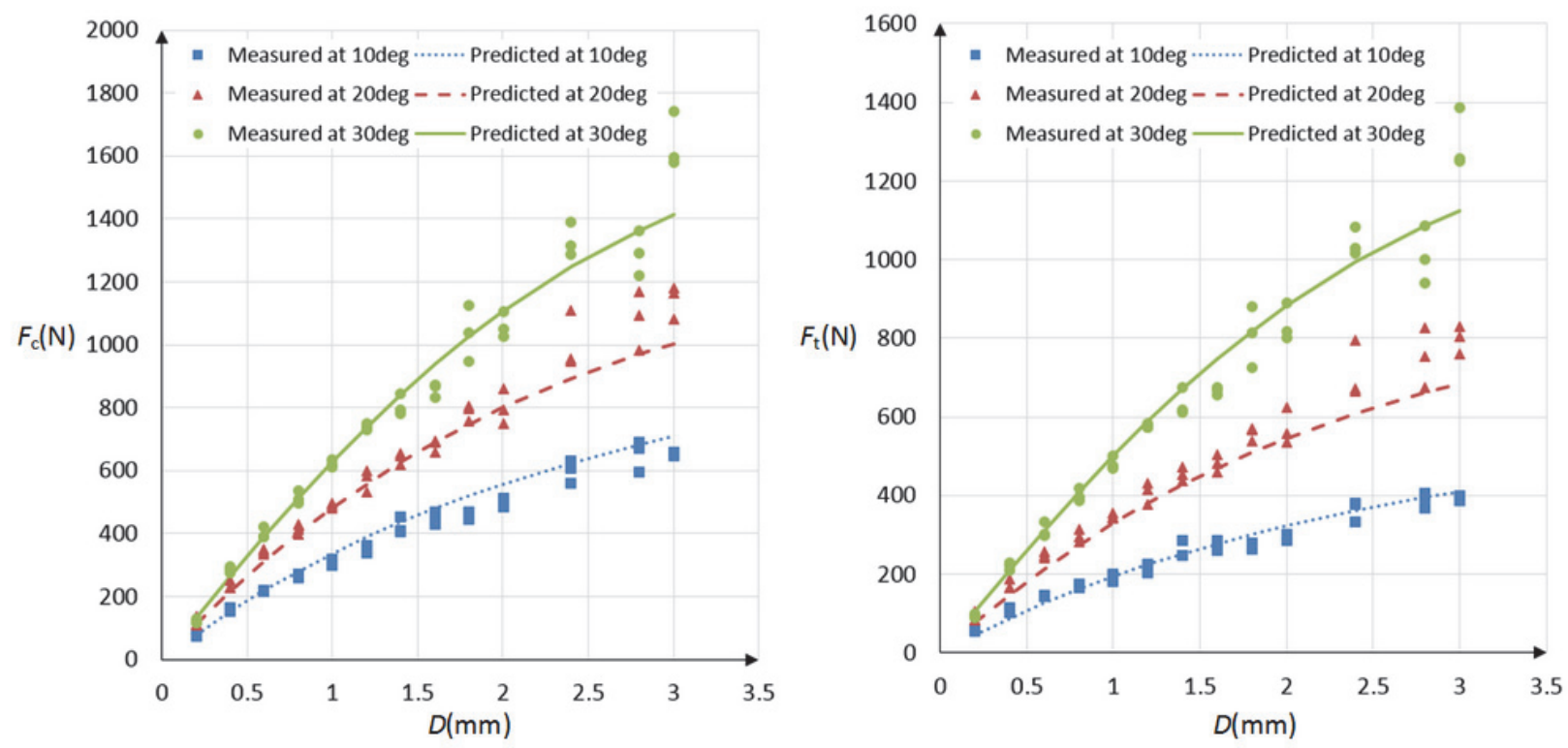

Fig. 14 Comparison of measured and predicted cutting and thrust forces in linear cutting of Indiana limestone with three levels of rake angles: $10^{\circ}$ in blue, $20^{\circ}$ in red, and $30^{\circ}$ in green 


\section{Discussion}

\subsection{Force responses}

As shown in Fig. 14, the average cutting and thrust forces increase non-linearly from very small $(\sim 0.2$ $\mathrm{mm})$ to quite large depths of cut $(\sim 3 \mathrm{~mm})$ values. However, the increase of the average force responses can be approximated with a linear trend for depths of cut below $1 \mathrm{~mm}$. This is in good agreement with other researchers' experimental findings [25, 38, 45]. Above a $1 \mathrm{~mm}$ depth of cut, the chunk-like chips due to the chipping action become more and more noticeable. Since the chipping mode induces cracks to form chips, while the crushing mode has to smash the rock into powdery chips, the chipping mode requires less energy and force than the crushing mode. Therefore, the average force responses become concave downward with an increase in the depth of cut.

\subsection{Chipping-Crushing Transition Angle}

As defined in Section 2.3, the chipping-crushing transition angle $\omega$ will determine the portion of the TCZ which induces the chipping mode. The decrease of $\omega$ results in an increase of the chipping mode, which in turn, leads to an increase in the size of the chip. Figure 15 gives a better illustration of how $\omega$ relates to the chip formation phenomena in the linear rock cutting process. $\omega$ was observed to decrease with the increase of the depth of cut, which means the chipping mode becomes more noticeable at deeper cuts.

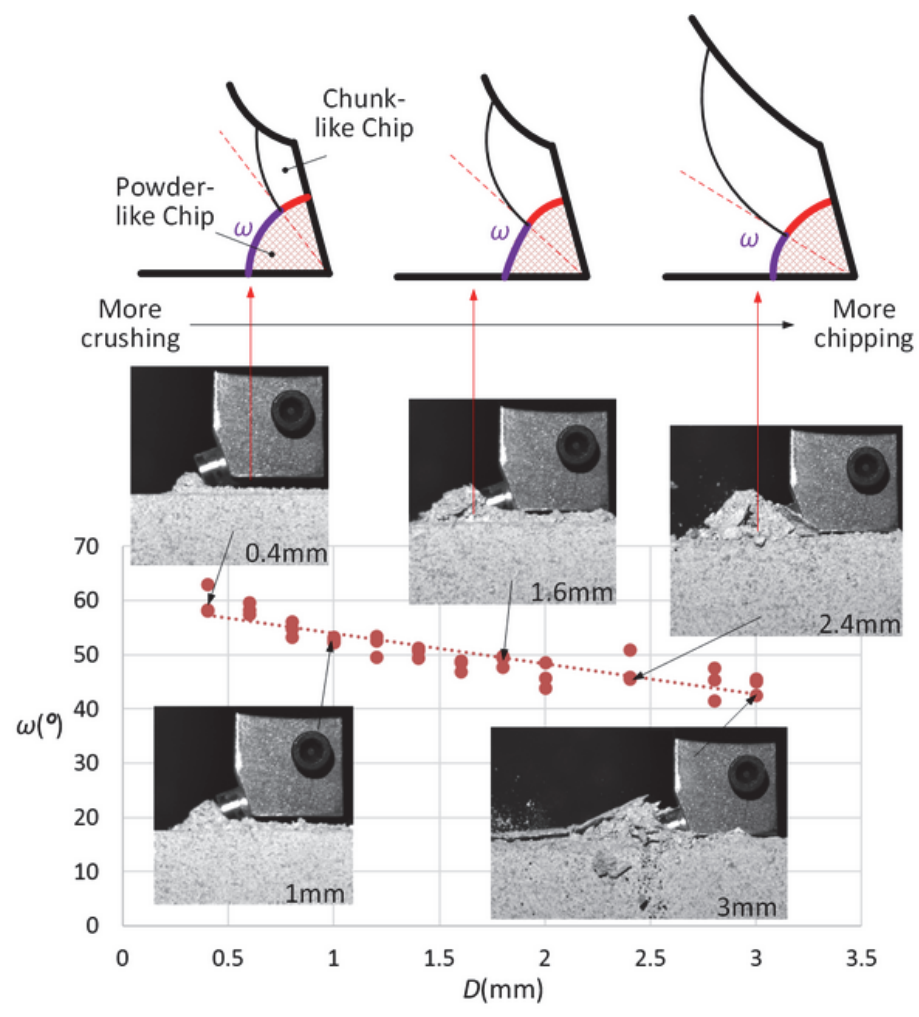

Fig. 15 Effect of the chipping-crushing transition angle on chip formation for a rake angle of $20^{\circ}$

As shown in Fig. 15, the rock fails with crushed and powdery chips for shallow cuts while both powdery chips and chunk-like chips are formed simultaneously for deep cuts. Moreover, the chip size increases with the increase of the depth of cut. As mentioned in Section 2.1, powdery and chunk-like chips are considered to be formed due to the crushing and chipping failure modes respectively. The above 
experimental observations indicate that the chipping mode becomes more and more obvious and dominant from small to big depths of cut. Therefore, the experimental observations are in good agreement with the physical meaning of $\omega$.

\subsection{Tip Crushing Ratio}

Based on Eq. (34), the tip crushing ratio $M$ can be expressed by:

$$
M=D_{\mathrm{TCZ}} / D
$$

According to Eq. (29), the required cutting force can be obtained by:

$$
F_{c}=\sigma_{c} w D M=\sigma_{c} A_{c} M
$$

where $A_{\mathrm{c}}$ is the cross-section of the cutting area along the cutting speed direction. Based on Eq. (44), with predefined $A_{\mathrm{c}}$ and $\sigma_{\mathrm{c}}, M$ will be the only parameter which influences the cutting force. The increase of $M$ will linearly increase the cutting force. Based on the geometrical relationships in Fig. 5 and the angle relationship in Eq. (31), the range of $\omega$ can be determined by:

$$
0 \leq \omega \leq 90^{\circ}-\gamma
$$

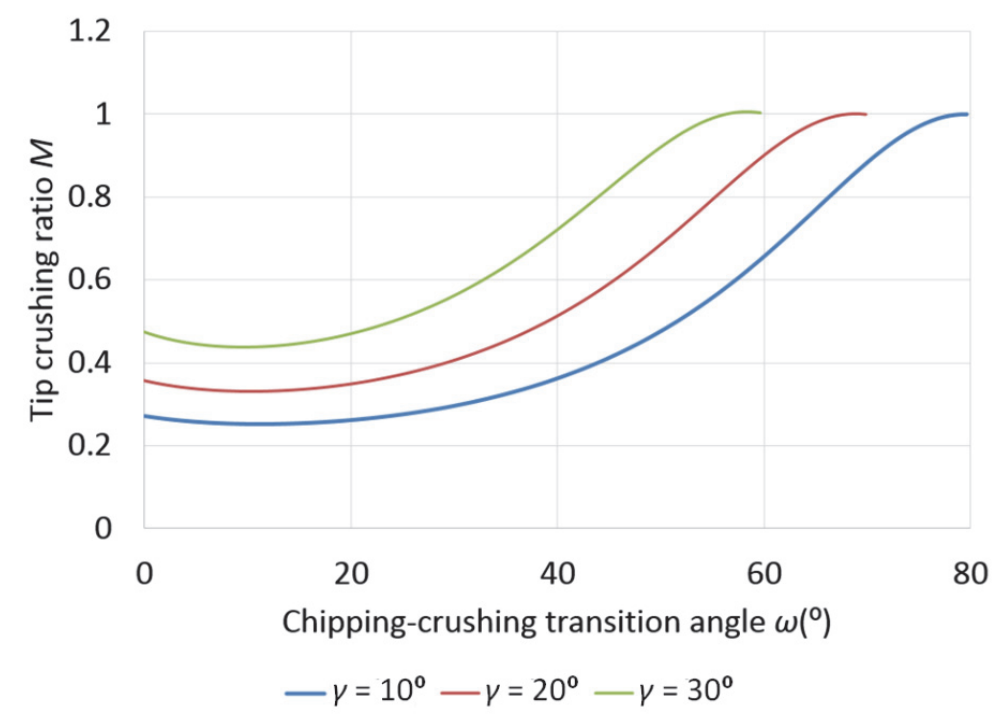

Fig. 16 Relationship between the tip crushing ratio and chipping-crushing transition angle

According to Eqs. (34), (35) and (45), the change of $M$ with respect to the chipping-crushing transition angle $\omega$ can be shown by the plots in Fig. 16. As shown in Fig. 16, the increase of the rake angle $\gamma$ decreases the maximal value of $\omega$ but increases the possible values of $M$. $M$ was observed to approach 1 when $\omega$ reaches its maximal value. From the physical perspective, the increase of $\omega$ decreases the chipping action but increases the crushing action in the whole rock removal process, which, in turn, increases the required cutting force. According to Eq. (44), $M$ is proportional to the cutting force. To this 
end, $M$ increases as $\omega$ increases. Moreover, the increase of the rake angle will increase the cutting force as shown in Fig. 14, which will also increase $M$.

\section{Conclusions}

The following conclusions can be drawn from this work:

(i) The rock removal mechanism in the rock cutting process includes crushing and chipping failure modes. Crushed and powdery chips are attributed to the crushing mode while chunk-like chips are formed due to the chipping mode. Chipping of rock has been concluded to be caused by either tensile or shear stresses by different researchers. However, no agreement has been reached.

(ii) This paper has developed a new cutting theory to describe both the crushing and chipping failures involved in the linear rock cutting process. A rock crushing zone, namely the Tip Crushing Zone (TCZ) that is formed beneath the cutter's rake face was defined. Cracks were assumed to initiate on the boundary of the TCZ and propagate towards the free surface of the virgin rock in order to form a chip. The chipping failure was considered to be caused by tensile breakage of the rock. Based on the equilibrium of moments on the chip and the minimum chipping energy principle, an analytical expression of the force responses was derived.

(iii) Several parameters were defined in the theory with clear physical meaning. The crack inclination angle indicates the direction of the crack's propagation path; the chipping-crushing transition angle characterizes the transition boundary between the chipping and crushing affected zones; the tip crushing ratio reflects how deep the TCZ is compared to the defined depth of cut; and the mean friction angle between the rock and the cutter's rake face relates the cutting and thrust forces.

(iv) A rock cutting testbed was developed and utilized to conduct linear rock cutting tests. Indiana limestone was selected as the testing rock sample. Cutting tests with 3 levels of rake angles and 13 levels of depths of cut were designed and conducted to provide experimental evaluation of the theory.

(v) Two characteristic parameters, i.e., the chipping-crushing transition angle and the mean friction angle between the rock and the cutter's rake face were calibrated based on experimental data. The chippingcrushing transition angle was found to be linear with respect to the depth of cut while the mean friction angle was found to be linear with respect to the rake angle.

(vi) The predicted cutting and thrust forces have shown a non-linear relationship between the force responses and the depth of cut. The forces were approximately proportional to the depth of cut for shallow cutting tests, i.e., for depths of cut below $1 \mathrm{~mm}$ for Indiana limestone, which are in good agreement with others' work.

(vii) The chip formation phenomena were found in good agreement with the theoretical predictions from the physical point of view. 


\section{$1^{\text {st }}$ Revision of the Manuscript: Confidential}

\section{Acknowledgments}

This work is supported by a National Science Foundation (NSF) collaborative research Grant No. CMMI1301127. The authors would like to thank Dr. John W. Rudnicki for his insightful review of the manuscript.

\section{References}

[1] G. Bruton, R. Crockett, M. Taylor, D. DenBoer, J. Lund, C. Fleming, R. Ford, G. Garcia, A. White, PDC bit technology for the 21st century, Oilfield Review, 26 (2014).

[2] D. Che, J. Smith, K. Ehmann, Finite element study of the cutting mechanics of the three dimensional rock turning process, in: ASME 2015 International Manufacturing Sciencen and Engineering Conference, ASME, Charlotte, North Carolina, USA, 2015, pp. MSEC2015-9249.

[3] D. Che, K. Ehmann, J. Cao, Analytical modeling of heat transfer in polycrystalline diamond compact cutters in rock turning processes, Journal of Manufacturing Science and Engineering, 137 (2015) 031005.

[4] D. Che, P. Han, P. Guo, K. Ehmann, Issues in polycrystalline diamond compact cutter-rock interaction from a metal machining point of view - part II: bit performance and rock cutting mechanics, Journal of Manufacturing Science and Engineering, 134 (2012) 064002.

[5] D. Che, P. Han, P. Guo, K. Ehmann, Issues in polycrystalline diamond compact cutter-rock interaction from a metal machining point of view - part I: temperature, stresses, and forces, Journal of Manufacturing Science and Engineering, 134 (2012) 064001.

[6] L.L. Mishnaevsky, Physical mechanisms of hard rock fragmentation under mechanical loading: A review, International Journal of Rock Mechanics and Mining Sciences \& Geomechanics Abstracts, 32 (1995) 763-766.

[7] I. Evans, A theory of the basic mechanics of coal ploughing, in: Proceedings of the international symposium on mining research, Univ. of Missouri at Rolla, Pergamon Press, 1962, pp. 761-798.

[8] M. Hood, H. Alehossein, A development in rock cutting technology, International Journal of Rock Mechanics and Mining Sciences, 37 (2000) 297-305.

[9] R. Goodrich, High pressure rotary drilling machines, Bull, 94 (1956).

[10] D. Zeuch, J. Finger, Rock breakage mechanisms with a PDC cutter, in: SPE Annual Technical Conference and Exhibition, Society of Petroleum Engineers, 1985.

[11] L.L. Mishnaevsky, Investigation of the cutting of brittle materials, International Journal of Machine Tools and Manufacture, 34 (1994) 499-505.

[12] H. Guo, N.I. Aziz, L.C. Schmidt, Rock cutting study using linear elastic fracture mechanics, Engineering Fracture Mechanics, 41 (1992) 771-778.

[13] T. Richard, Determination of Rock Strength from Cutting Tests, University of Minnesota, 1999.

[14] N.G. Yilmaz, M. Yurdakul, R. Goktan, Prediction of radial bit cutting force in high-strength rocks using multiple linear regression analysis, International Journal of Rock Mechanics and Mining Sciences, 44 (2007) 962-970.

[15] R. Goktan, N.G. Yilmaz, A new methodology for the analysis of the relationship between rock brittleness index and drag pick cutting efficiency, Journal - South African Institute of Mining and Metallurgy, 105 (2005) 727.

[16] K. Liu, X.P. Li, S.Y. Liang, The mechanism of ductile chip formation in cutting of brittle materials, The International Journal of Advanced Manufacturing Technology, 33 (2007) 875-884.

[17] Y. Nishimatsu, The mechanics of rock cutting, International Journal of Rock Mechanics and Mining Sciences, 9 (1972) 261 270.

[18] Y. Nishimatsu, Theories of rock cutting, in: J.A. Hudson (Ed.) Comprehensive Rock Engineering : Principles, Practice \& Projects, Oxford ; New York : Pergamon Press 1993.

[19] C.J. Durrand, M.R. Skeem, R.B. Crockett, D.R. Hall, Super-hard, thick, shaped PDC cutters for hard rock drilling: development and test results, in: 35th Workshop on Geothermal Reservoir Engineering, Stanford, California.

[20] D. Che, K. Ehmann, Polycrystalline diamond turning of rock, in: ASME 2013 International Manufacturing Science and Engineering Conference, Madison, Wisconsin, USA, 2013, pp. MSEC2013-1127.

[21] D. Che, K. Ehmann, Experimental study of force responses in polycrystalline diamond face turning of rock, International Journal of Rock Mechanics and Mining Sciences, 72 (2014) 80-91.

[22] C. Fairhurst, W. Lacabanne, Some principles and developments in hard rock drilling techniques, (1956).

[23] E. Detournay, P. Defourny, A phenomenological model for the drilling action of drag bits, International Journal of Rock Mechanics and Mining Sciences \& Geomechanics Abstracts, 29 (1992) 13-23.

[24] B. Tiryaki, Evaluation of the indirect measures of rock brittleness and fracture toughness in rock cutting, JOURNALSOUTH AFRICAN INSTITUTE OF MINING AND METALLURGY, 106 (2006) 407.

[25] T. Richard, F. Dagrain, E. Poyol, E. Detournay, Rock strength determination from scratch tests, Engineering Geology, 147 (2012) 91-100.

[26] H. Guo, N.I. Aziz, L.C. Schmidt, Linear elastic crack tip modelling by the displacement discontinuity method, Engineering Fracture Mechanics, 36 (1990) 933-943.

[27] W. Yan, Single PDC cutter force modeling for hard rock cutting, in: Petroleum and Natural Gas Engineering, New Mexico Institute of Mining and Technology Socorro, New Mexico, 1997. 


\section{$1^{\text {st }}$ Revision of the Manuscript: Confidential}

[28] X.C. Tan, S.Q. Kou, P.A. Lindqvist, Application of the DDM and fracture mechanics model on the simulation of rock breakage by mechanical tools, Engineering Geology, 49 (1998) 277-284.

[29] R.K. Zipf, Z.T. Bieniawski, Estimating the crush zone size under a cutting tool in coal, International Journal of Mining and Geological Engineering, 6 (1988) 279-295.

[30] H. Huang, B. Lecampion, E. Detournay, Discrete element modeling of tool-rock interaction I: rock cutting, International Journal for Numerical and Analytical Methods in Geomechanics, 37 (2013) 1913-1929.

[31] J. Xue, Y. Xia, Z. Ji, X. Zhou, Soft rock cutting mechanics model of TBM cutter and experimental research, in: M. Xie, Y. Xiong, C. Xiong, H. Liu, Z. Hu (Eds.) Intelligent Robotics and Applications, Springer Berlin Heidelberg, 2009, pp. 383391.

[32] M.E. Merchant, Basic mechanics of the metal-cutting process, Journal of Applied Mechanics, 11 (1944) 168-175.

[33] M.E. Merchant, Mechanics of the metal cutting process. I. Orthogonal cutting and a type 2 chip, Journal of Applied Physics, 16 (1945) 267-275.

[34] V. Prakash, Rock cutting theory for PDC cutters, in, Kansas State University, 1982.

[35] K.E. Rånman, A model describing rock cutting with conical picks, Rock mechanics and rock engineering, 18 (1985) 131140.

[36] I. Evans, The force required to cut coal with blunt wedges, International Journal of Rock Mechanics and Mining Sciences \& Geomechanics Abstracts, 2 (1965) 1-12.

[37] G. Boothroyd, W.A. Knight, Fundamentals of machining and machine tools, Marcel Dekker Inc., New York, 1989.

[38] T. Richard, Determination of rock strength from cutting tests, in: Graduate School, University of Minnesota, 1999.

[39] A. Scheidegger, Principles of Geodynamics, Springer-Verlag, 1982.

[40] D. Che, P. Han, B. Peng, K.F. Ehmann, Finite element study on chip formation and force response in two-dimensional orthogonal cutting of rock, in: ASME 2014 International Manufacturing Science and Engineering Conference, 2014, pp. MSEC2014-3952.

[41] D. Che, W. Zhang, K. Ehmann, Chip Formation and Force Responses in Linear Rock Cutting: An Experimental Study, Journal of Manufacturing Science and Engineering, 139 (2016) 011011.

[42] D. Che, W. Zhang, K.F. Ehmann, Rock Cutter Interactions in Linear Rock Cutting, in: ASME 201611 th International Manufacturing Science and Engineering Conference, American Society of Mechanical Engineers, 2016, pp. MSEC20168510 .

[43] Kocurek Industries, Indiana limestone, in, 2015.

[44] Indiana Limestone Institue, Indiana limestone handbook, in, 2007.

[45] M.C. Jaime, I.K. Gamwo, D.K. Lyons, J.S. Lin, Finite element modeling of rock cutting, in: 44th US Rock Mechanics Symposium and 5th U.S.-Canada Rock Mechanics Symposium, American Rock Mechanics Association, Salt Lake City, UT, 2010, pp. 10-231. 
$1^{\text {st }}$ Revision of the Manuscript: Confidential

\section{Appendix}

Table A.1: Process parameters and corresponding force responses for the experiments

\begin{tabular}{|c|c|c|c|c|c|c|c|c|c|}
\hline $\begin{array}{l}\text { Test } \\
\text { No. }\end{array}$ & $\begin{array}{c}\text { Rake } \\
\text { angle } \\
\left({ }^{\circ}\right)\end{array}$ & $\begin{array}{l}\text { Depth } \\
\text { of cut } \\
(\mathrm{mm})\end{array}$ & $\begin{array}{l}\text { Average } \\
\text { cutting } \\
\text { force }(\mathrm{N})\end{array}$ & $\begin{array}{c}\text { Average } \\
\text { thrust } \\
\text { force }(\mathrm{N})\end{array}$ & $\begin{array}{l}\text { Average } \\
\text { side force } \\
\text { (N) }\end{array}$ & $\begin{array}{l}\text { Predicted } \\
\text { cutting } \\
\text { force }(\mathrm{N})\end{array}$ & $\begin{array}{l}\text { Predicted } \\
\text { thrust } \\
\text { force }(N)\end{array}$ & $\begin{array}{l}\text { Cutting force } \\
\text { prediction } \\
\text { error }(\%)\end{array}$ & $\begin{array}{c}\text { Thrust force } \\
\text { prediction } \\
\text { error }(\%)\end{array}$ \\
\hline 1 & 10 & 0.2 & 83.08 & 61.94 & -0.06 & 78.99 & 45.68 & $5 \%$ & $26 \%$ \\
\hline 2 & 10 & 0.2 & 80.64 & 61.57 & -5.37 & 78.99 & 45.68 & $2 \%$ & $26 \%$ \\
\hline 3 & 10 & 0.2 & 72.49 & 53.27 & -0.49 & 78.99 & 45.68 & $9 \%$ & $14 \%$ \\
\hline 4 & 10 & 0.4 & 162.72 & 113.83 & 0.48 & 151.62 & 87.68 & $7 \%$ & $23 \%$ \\
\hline 5 & 10 & 0.4 & 158.14 & 109.86 & -0.49 & 151.62 & 87.68 & $4 \%$ & $20 \%$ \\
\hline 6 & 10 & 0.4 & 151.58 & 102.61 & -1.83 & 151.62 & 87.68 & $0 \%$ & $15 \%$ \\
\hline 7 & 10 & 0.6 & 219.03 & 141.86 & 1.56 & 218.33 & 126.26 & $0 \%$ & $11 \%$ \\
\hline 8 & 10 & 0.6 & 217.49 & 143.39 & -1.47 & 218.33 & 126.26 & $0 \%$ & $12 \%$ \\
\hline 9 & 10 & 0.6 & 217.02 & 145.43 & -2.05 & 218.33 & 126.26 & $1 \%$ & $13 \%$ \\
\hline 10 & 10 & 0.8 & 265.19 & 166.72 & 0.90 & 279.59 & 161.68 & $5 \%$ & $3 \%$ \\
\hline 11 & 10 & 0.8 & 272.71 & 175.97 & -1.67 & 279.59 & 161.68 & $3 \%$ & $8 \%$ \\
\hline 12 & 10 & 0.8 & 259.74 & 165.02 & -2.94 & 279.59 & 161.68 & $8 \%$ & $2 \%$ \\
\hline 13 & 10 & 1 & 299.76 & 182.36 & 1.56 & 335.85 & 194.21 & $12 \%$ & $6 \%$ \\
\hline 14 & 10 & 1 & 317.28 & 198.98 & 0.43 & 335.85 & 194.21 & $6 \%$ & $2 \%$ \\
\hline 15 & 10 & 1 & 317.33 & 196.66 & -2.32 & 335.85 & 194.21 & $6 \%$ & $1 \%$ \\
\hline 16 & 10 & 1.2 & 336.33 & 203.65 & 0.36 & 387.54 & 224.11 & $15 \%$ & $10 \%$ \\
\hline 17 & 10 & 1.2 & 358.21 & 219.98 & -0.61 & 387.54 & 224.11 & $8 \%$ & $2 \%$ \\
\hline 18 & 10 & 1.2 & 363.48 & 225.44 & -5.63 & 387.54 & 224.11 & $7 \%$ & $1 \%$ \\
\hline 19 & 10 & 1.4 & 405.79 & 247.58 & -1.25 & 435.10 & 251.61 & $7 \%$ & $2 \%$ \\
\hline 20 & 10 & 1.4 & 411.00 & 248.89 & 1.27 & 435.10 & 251.61 & $6 \%$ & $1 \%$ \\
\hline 21 & 10 & 1.4 & 454.36 & 285.99 & -0.91 & 435.10 & 251.61 & $4 \%$ & $12 \%$ \\
\hline 22 & 10 & 1.6 & 428.18 & 259.22 & 1.89 & 478.91 & 276.95 & $12 \%$ & $7 \%$ \\
\hline 23 & 10 & 1.6 & 450.22 & 275.51 & -2.59 & 478.91 & 276.95 & $6 \%$ & $1 \%$ \\
\hline 24 & 10 & 1.6 & 468.33 & 285.17 & 3.61 & 478.91 & 276.95 & $2 \%$ & $3 \%$ \\
\hline 25 & 10 & 1.8 & 467.22 & 279.96 & 4.04 & 519.37 & 300.34 & $11 \%$ & $7 \%$ \\
\hline 26 & 10 & 1.8 & 463.06 & 279.14 & -0.14 & 519.37 & 300.34 & $12 \%$ & $8 \%$ \\
\hline 27 & 10 & 1.8 & 445.33 & 263.60 & -3.63 & 519.37 & 300.34 & $17 \%$ & $14 \%$ \\
\hline 28 & 10 & 2 & 483.24 & 286.04 & 0.66 & 556.81 & 321.99 & $15 \%$ & $13 \%$ \\
\hline 29 & 10 & 2 & 484.57 & 287.55 & 2.48 & 556.81 & 321.99 & $15 \%$ & $12 \%$ \\
\hline 30 & 10 & 2 & 510.99 & 300.48 & -0.98 & 556.81 & 321.99 & $9 \%$ & $7 \%$ \\
\hline 31 & 10 & 2.4 & 605.40 & 377.26 & 2.42 & 623.94 & 360.82 & $3 \%$ & $4 \%$ \\
\hline 32 & 10 & 2.4 & 632.51 & 381.87 & 10.29 & 623.94 & 360.82 & $1 \%$ & $6 \%$ \\
\hline 33 & 10 & 2.4 & 558.52 & 333.43 & -2.03 & 623.94 & 360.82 & $12 \%$ & $8 \%$ \\
\hline 34 & 10 & 2.8 & 670.45 & 395.73 & -0.27 & 682.63 & 394.75 & $2 \%$ & $0 \%$ \\
\hline 35 & 10 & 2.8 & 594.71 & 367.41 & -1.60 & 682.63 & 394.75 & $15 \%$ & $7 \%$ \\
\hline 36 & 10 & 2.8 & 690.65 & 406.98 & 7.07 & 682.63 & 394.75 & $1 \%$ & $3 \%$ \\
\hline 37 & 10 & 3 & 658.31 & 398.35 & 3.20 & 709.44 & 410.26 & $8 \%$ & $3 \%$ \\
\hline 38 & 10 & 3 & 645.94 & 386.56 & 4.46 & 709.44 & 410.26 & $10 \%$ & $6 \%$ \\
\hline 39 & 10 & 3 & 656.86 & 387.68 & 1.85 & 709.44 & 410.26 & $8 \%$ & $6 \%$ \\
\hline 40 & 20 & 0.2 & 135.96 & 105.57 & -2.33 & 110.91 & 75.65 & $18 \%$ & $28 \%$ \\
\hline 41 & 20 & 0.2 & 111.26 & 84.12 & -2.86 & 110.91 & 75.65 & $0 \%$ & $10 \%$ \\
\hline 42 & 20 & 0.2 & 110.79 & 82.15 & 1.96 & 110.91 & 75.65 & $0 \%$ & $8 \%$ \\
\hline 43 & 20 & 0.4 & 250.07 & 186.91 & -1.44 & 214.61 & 146.39 & $14 \%$ & $22 \%$ \\
\hline 44 & 20 & 0.4 & 226.86 & 165.23 & 0.44 & 214.61 & 146.39 & $5 \%$ & $11 \%$ \\
\hline 45 & 20 & 0.4 & 226.32 & 165.08 & -2.12 & 214.61 & 146.39 & $5 \%$ & $11 \%$ \\
\hline 46 & 20 & 0.6 & 351.66 & 255.92 & -6.46 & 310.99 & 212.13 & $12 \%$ & $17 \%$ \\
\hline 47 & 20 & 0.6 & 334.03 & 241.17 & -2.78 & 310.99 & 212.13 & $7 \%$ & $12 \%$ \\
\hline 48 & 20 & 0.6 & 341.34 & 247.22 & -4.06 & 310.99 & 212.13 & $9 \%$ & $14 \%$ \\
\hline 49 & 20 & 0.8 & 430.02 & 312.97 & -5.85 & 400.09 & 272.90 & $7 \%$ & $13 \%$ \\
\hline 50 & 20 & 0.8 & 396.23 & 281.76 & -1.85 & 400.09 & 272.90 & $1 \%$ & $3 \%$ \\
\hline 51 & 20 & 0.8 & 413.76 & 296.31 & -7.09 & 400.09 & 272.90 & $3 \%$ & $8 \%$ \\
\hline
\end{tabular}


$1^{\text {st }}$ Revision of the Manuscript: Confidential

\begin{tabular}{|c|c|c|c|c|c|c|c|c|c|}
\hline 52 & 20 & 1 & 496.44 & 356.43 & -7.45 & 482.13 & 328.86 & $3 \%$ & $8 \%$ \\
\hline 53 & 20 & 1 & 481.71 & 341.29 & 1.04 & 482.13 & 328.86 & $0 \%$ & $4 \%$ \\
\hline 54 & 20 & 1 & 487.51 & 345.64 & -3.82 & 482.13 & 328.86 & $1 \%$ & $5 \%$ \\
\hline 55 & 20 & 1.2 & 598.92 & 429.59 & -13.52 & 557.42 & 380.22 & $7 \%$ & $11 \%$ \\
\hline 56 & 20 & 1.2 & 533.40 & 376.23 & -1.33 & 557.42 & 380.22 & $5 \%$ & $1 \%$ \\
\hline 57 & 20 & 1.2 & 582.13 & 414.01 & -9.03 & 557.42 & 380.22 & $4 \%$ & $8 \%$ \\
\hline 58 & 20 & 1.4 & 654.88 & 471.68 & -13.57 & 626.34 & 427.23 & $4 \%$ & $9 \%$ \\
\hline 59 & 20 & 1.4 & 618.13 & 437.43 & -4.34 & 626.34 & 427.23 & $1 \%$ & $2 \%$ \\
\hline 60 & 20 & 1.4 & 644.52 & 454.32 & -0.39 & 626.34 & 427.23 & $3 \%$ & $6 \%$ \\
\hline 61 & 20 & 1.6 & 695.40 & 502.36 & -11.37 & 689.33 & 470.20 & $1 \%$ & $6 \%$ \\
\hline 62 & 20 & 1.6 & 690.31 & 483.26 & -0.16 & 689.33 & 470.20 & $0 \%$ & $3 \%$ \\
\hline 63 & 20 & 1.6 & 656.44 & 460.45 & 2.43 & 689.33 & 470.20 & $5 \%$ & $2 \%$ \\
\hline 64 & 20 & 1.8 & 797.58 & 569.10 & -8.22 & 746.87 & 509.44 & $6 \%$ & $10 \%$ \\
\hline 65 & 20 & 1.8 & 757.44 & 537.99 & -2.71 & 746.87 & 509.44 & $1 \%$ & $5 \%$ \\
\hline 66 & 20 & 1.8 & 804.86 & 566.53 & -6.13 & 746.87 & 509.44 & $7 \%$ & $10 \%$ \\
\hline 67 & 20 & 2 & 861.33 & 625.22 & -16.20 & 799.43 & 545.29 & $7 \%$ & $13 \%$ \\
\hline 68 & 20 & 2 & 790.79 & 556.60 & 1.49 & 799.43 & 545.29 & $1 \%$ & $2 \%$ \\
\hline 69 & 20 & 2 & 749.20 & 535.57 & 3.79 & 799.43 & 545.29 & $7 \%$ & $2 \%$ \\
\hline 70 & 20 & 2.4 & 1109.94 & 795.27 & -30.78 & 891.50 & 608.10 & $20 \%$ & $24 \%$ \\
\hline 71 & 20 & 2.4 & 956.47 & 672.38 & -30.74 & 891.50 & 608.10 & $7 \%$ & $10 \%$ \\
\hline 72 & 20 & 2.4 & 946.48 & 664.46 & -10.97 & 891.50 & 608.10 & $6 \%$ & $8 \%$ \\
\hline 73 & 20 & 2.8 & 982.15 & 675.65 & -28.50 & 969.16 & 661.07 & $1 \%$ & $2 \%$ \\
\hline 74 & 20 & 2.8 & 1094.33 & 752.44 & -25.32 & 969.16 & 661.07 & $11 \%$ & $12 \%$ \\
\hline 75 & 20 & 2.8 & 1167.77 & 825.26 & -3.17 & 969.16 & 661.07 & $17 \%$ & $20 \%$ \\
\hline 76 & 20 & 3 & 1081.51 & 760.18 & -11.98 & 1003.62 & 684.57 & $7 \%$ & $10 \%$ \\
\hline 77 & 20 & 3 & 1179.45 & 829.53 & 11.67 & 1003.62 & 684.57 & $15 \%$ & $17 \%$ \\
\hline 78 & 20 & 3 & 1163.67 & 804.72 & 11.24 & 1003.62 & 684.57 & $14 \%$ & $15 \%$ \\
\hline 79 & 30 & 0.2 & 117.59 & 91.51 & 1.65 & 131.53 & 104.84 & $12 \%$ & $15 \%$ \\
\hline 80 & 30 & 0.2 & 118.30 & 90.13 & 0.91 & 131.53 & 104.84 & $11 \%$ & $16 \%$ \\
\hline 81 & 30 & 0.2 & 127.75 & 99.56 & 2.04 & 131.53 & 104.84 & $3 \%$ & $5 \%$ \\
\hline 82 & 30 & 0.4 & 285.27 & 222.76 & -2.37 & 261.65 & 208.54 & $8 \%$ & $6 \%$ \\
\hline 83 & 30 & 0.4 & 273.03 & 210.83 & -1.30 & 261.65 & 208.54 & $4 \%$ & $1 \%$ \\
\hline 84 & 30 & 0.4 & 294.48 & 228.99 & 2.83 & 261.65 & 208.54 & $11 \%$ & $9 \%$ \\
\hline 85 & 30 & 0.6 & 421.75 & 334.35 & -5.00 & 388.71 & 309.82 & $8 \%$ & $7 \%$ \\
\hline 86 & 30 & 0.6 & 388.97 & 297.13 & 0.17 & 388.71 & 309.82 & $0 \%$ & $4 \%$ \\
\hline 87 & 30 & 0.6 & 390.19 & 302.01 & 2.57 & 388.71 & 309.82 & $0 \%$ & $3 \%$ \\
\hline 88 & 30 & 0.8 & 535.14 & 418.83 & 1.48 & 511.29 & 407.52 & $4 \%$ & $3 \%$ \\
\hline 89 & 30 & 0.8 & 497.26 & 385.23 & -1.39 & 511.29 & 407.52 & $3 \%$ & $6 \%$ \\
\hline 90 & 30 & 0.8 & 509.55 & 395.05 & 6.75 & 511.29 & 407.52 & $0 \%$ & $3 \%$ \\
\hline 91 & 30 & 1 & 619.86 & 476.40 & -3.38 & 628.23 & 500.72 & $1 \%$ & $5 \%$ \\
\hline 92 & 30 & 1 & 635.49 & 500.46 & -0.81 & 628.23 & 500.72 & $1 \%$ & $0 \%$ \\
\hline 93 & 30 & 1 & 610.05 & 468.49 & 4.90 & 628.23 & 500.72 & $3 \%$ & $7 \%$ \\
\hline 94 & 30 & 1.2 & 730.58 & 574.93 & -2.05 & 738.62 & 588.71 & $1 \%$ & $2 \%$ \\
\hline 95 & 30 & 1.2 & 748.93 & 583.90 & 0.52 & 738.62 & 588.71 & $1 \%$ & $1 \%$ \\
\hline 96 & 30 & 1.2 & 738.28 & 572.92 & 7.95 & 738.62 & 588.71 & $0 \%$ & $3 \%$ \\
\hline 97 & 30 & 1.4 & 842.86 & 673.49 & 6.08 & 841.89 & 671.01 & $0 \%$ & $0 \%$ \\
\hline 98 & 30 & 1.4 & 782.21 & 612.12 & -3.98 & 841.89 & 671.01 & $8 \%$ & $10 \%$ \\
\hline 99 & 30 & 1.4 & 793.16 & 616.30 & 9.43 & 841.89 & 671.01 & $6 \%$ & $9 \%$ \\
\hline 100 & 30 & 1.6 & 833.33 & 655.15 & -1.92 & 937.68 & 747.36 & $13 \%$ & $14 \%$ \\
\hline 101 & 30 & 1.6 & 866.54 & 668.23 & -14.70 & 937.68 & 747.36 & $8 \%$ & $12 \%$ \\
\hline 102 & 30 & 1.6 & 873.52 & 675.68 & 1.80 & 937.68 & 747.36 & $7 \%$ & $11 \%$ \\
\hline 103 & 30 & 1.8 & 1123.71 & 880.01 & 4.28 & 1025.91 & 817.68 & $9 \%$ & $7 \%$ \\
\hline 104 & 30 & 1.8 & 1039.00 & 812.28 & -9.99 & 1025.91 & 817.68 & $1 \%$ & $1 \%$ \\
\hline 105 & 30 & 1.8 & 947.29 & 725.45 & 16.55 & 1025.91 & 817.68 & $8 \%$ & $13 \%$ \\
\hline 106 & 30 & 2 & 1104.37 & 889.00 & 9.38 & 1106.68 & 882.06 & $0 \%$ & $1 \%$ \\
\hline 107 & 30 & 2 & 1050.24 & 817.28 & -13.24 & 1106.68 & 882.06 & $5 \%$ & $8 \%$ \\
\hline 108 & 30 & 2 & 1026.40 & 799.86 & 8.85 & 1106.68 & 882.06 & $8 \%$ & $10 \%$ \\
\hline 109 & 30 & 2.4 & 1288.37 & 1015.05 & -6.11 & 1247.06 & 993.95 & $3 \%$ & $2 \%$ \\
\hline 110 & 30 & 2.4 & 1387.82 & 1083.89 & -20.72 & 1247.06 & 993.95 & $10 \%$ & $8 \%$ \\
\hline 111 & 30 & 2.4 & 1314.65 & 1028.24 & 15.52 & 1247.06 & 993.95 & $5 \%$ & $3 \%$ \\
\hline 112 & 30 & 2.8 & 1217.76 & 941.20 & -2.22 & 1362.27 & 1085.78 & $12 \%$ & $15 \%$ \\
\hline
\end{tabular}


$1^{\text {st }}$ Revision of the Manuscript: Confidential

\begin{tabular}{c|cc|ccc|cc|cc}
\hline 113 & 30 & 2.8 & 1362.21 & 1084.69 & -53.24 & 1362.27 & 1085.78 & $0 \%$ & $0 \%$ \\
114 & 30 & 2.8 & 1290.86 & 999.24 & 9.02 & 1362.27 & 1085.78 & $6 \%$ & $9 \%$ \\
115 & 30 & 3 & 1740.73 & 1384.76 & 13.95 & 1411.79 & 1125.25 & $19 \%$ & $19 \%$ \\
116 & 30 & 3 & 1596.21 & 1257.56 & -44.73 & 1411.79 & 1125.25 & $12 \%$ & $11 \%$ \\
117 & 30 & 3 & 1578.68 & 1251.20 & -13.17 & 1411.79 & 1125.25 & $11 \%$ & $10 \%$ \\
\hline
\end{tabular}

